\title{
Monotone traveling waves for reaction-diffusion equations involving the curvature operator
}

\author{
Maurizio Garrione ${ }^{1}$ and Luís Sanchez $z^{2^{*}}$
}

\section{"Correspondence:}

Ifrodrigues@fc.ul.pt

${ }^{2}$ CMAF, Faculdade de Ciências da

Universidade de Lisboa, Avenida

Professor Gama Pinto, 2, Lisboa,

1649-003, Portugal

Full list of author information is

available at the end of the article

\begin{abstract}
We study the existence of monotone traveling waves $u(t, x)=u(x+c t)$, connecting two equilibria, for the reaction-diffusion PDE $u_{t}=\left(\frac{u_{x}}{\sqrt{1+u_{x}^{2}}}\right)_{x}+f(u)$. Assuming different forms for the reaction term $f(u)$ (among which we have the so-called types $A, B$, and $C$ ), we show that, concerning the admissible speeds, the situation presents both similarities and differences with respect to the classical case. We use a first order model obtained after a suitable change of variables. The model contains a singularity and therefore has some features which are not present in the case of linear diffusion. The technique used involves essentially shooting arguments and lower and upper solutions. Some numerical simulations are provided in order to better understand the features of the model.

MSC: $34 \mathrm{C} 37 ; 35 \mathrm{~K} 57 ; 34 \mathrm{~B} 18$
\end{abstract}

Keywords: traveling waves; lower and upper solutions; mean curvature operator

\section{Introduction}

In this paper, we will be interested in the existence of monotone heteroclinic solutions for a quasilinear variant of the scalar second order differential equation

$$
u^{\prime \prime}-c u^{\prime}+f(u)=0
$$

which arises in connection with the Fisher-Kolmogorov PDE

$$
u_{t}=u_{x x}+f(u)
$$

when searching for traveling waves with speed $c$, i.e., solutions having the form $u(t, x)=$ $u(x+c t)$. Equation (2) was originally proposed in 1937 by $\mathrm{R}$. Fisher, to model the 1dimensional spreading of an advantageous gene with frequency $u(t, x)$, and analyzed from the mathematical point of view by Kolmogorov, Petrovsky and Piskounov [1] (for this reason, it is briefly referred to as FKPP). From then on, it has found many different applications and variants in several fields, such as ecology, physiology, combustion, general phase transition problems.

The process described by (2) is a reaction-diffusion one, where the spatial diffusion is linear. The reaction term may have several forms, but we will always assume that it satisfies

\section{Springer}

๑) 2015 Garrione and Sanchez; licensee Springer. This is an Open Access article distributed under the terms of the Creative Commons Attribution License (http://creativecommons.org/licenses/by/4.0), which permits unrestricted use, distribution, and reproduction in any medium, provided the original work is properly credited. 
$f(0)=0=f(1)$, meaning that no reaction is present if the gene is completely spread or not spread at all into the population. Accordingly, we will be interested in monotone traveling waves connecting 0 (at $-\infty$ ) and 1 (at $+\infty$ ) and taking values strictly included between them, i.e., using the terminology in [2], the so-called front-type solutions. The existence of such connections turns out to be important since, for instance in the case of linear diffusion, under certain assumptions on the initial datum the solutions to the PDE (2) converge, for $t \rightarrow+\infty$, to suitable combinations of traveling wave-type solutions (see, e.g., $[3,4])$.

The linearity of the diffusion term, however, is not always appropriate to model a realistic phenomenon. Indeed, it has been observed that, referring to the dependence of the diffusion with respect to $\nabla u$, there may occur in some instances a kind of saturation effect for large $|\nabla u|$ (while a linear dependence in the gradient, for $\nabla u$ small, is more realistic). In such cases, it seems reasonable to replace $\Delta$ with a certain type of nonlinear diffusion in (1). Precisely, the model which we will investigate is given by the flux-saturated diffusion equation (for a pioneer model of this kind, see for instance [5])

$$
u_{t}=\left(\frac{u_{x}}{\sqrt{1+u_{x}^{2}}}\right)_{x}+f(u)
$$

where the diffusion process is governed by a mean curvature type operator (see [6, 7]). Wishing to search for monotone traveling waves $u(t, x)=u(x+c t)$ for (3), connecting the two equilibria 0 and 1 , we will thus study the problem

$$
\left\{\begin{array}{l}
\left(\frac{u^{\prime}}{\sqrt{1+u^{\prime 2}}}\right)^{\prime}-c u^{\prime}+f(u)=0, \\
u(-\infty)=0, \quad u(+\infty)=1 ; \quad u^{\prime}(t)>0 \quad \text { for every } t
\end{array}\right.
$$

the values of $c$ for which (4) has a solution will be called admissible speeds.

It is well known that in the classical case, namely for equation (1), different phenomena may arise, depending on the shape of $f$. In particular, if $f(u)>0$ for every $u \in] 0,1[$, then the admissible speeds form an unbounded interval $\left[c^{*},+\infty\left[\right.\right.$, where the value $c^{*}$ takes the name of critical speed. On the contrary, it suffices to have $f(u) \leq 0$ in a neighborhood of 0 to force the admissible speed, if any, to be unique, using a monotonicity argument (see Lemma 3.8 below in our quite general context; we will use the name critical speed also in this case). In any case, it is immediate to see that the existence of a positive admissible speed is possible only if $f(u)>0$ in a neighborhood of $u=1$.

Another elementary observation narrowing the range for the admissible speeds is related to the nature of the equilibria 0 and 1 when $f$ is differentiable: linearizing around them yields the eigenvalues

$$
\lambda_{1,2}(0)=\frac{-c \pm \sqrt{c^{2}-4 f^{\prime}(0)}}{2}, \quad \lambda_{1,2}(1)=\frac{-c \pm \sqrt{c^{2}-4 f^{\prime}(1)}}{2},
$$

and we see that $u=1$ is a saddle if $f^{\prime}(1)<0$, while $u=0$ is a spiral for $c^{2}<4 f^{\prime}(0)$ or an unstable node for $c^{2} \geq 4 f^{\prime}(0)$. Thus, if we want to find monotone traveling waves we will have to ask that $c^{2} \geq 4 f^{\prime}(0)$ (while no requirements need to be done on $f^{\prime}(1)$, apart from its negative sign). Notice, however, that we can state results about (4) without differentiability assumptions; see Sections 2 and 3. 
We will study the features of the set of the admissible speeds for (4) for different classes of reaction terms, and provide, in some cases, a way of computing the critical speed. We shall see that our problem presents both similarities and differences with respect to the FKPP equation. The most significant feature, in particular, is the appearance here of a singularity (see Section 2) which makes the model more delicate to deal with than in the classical case, requiring some restrictions which are peculiar of (4) in order to find a regular solution. Indeed, elementary arguments show that a classical solution not always exists already for $c=0$, and one should in principle admit discontinuous solutions which satisfy the equation in a suitable weak sense, as was observed, e.g., in $[8,9]$. We remark that recent efforts have been devoted to the study of periodic and Dirichlet boundary value problems associated with (4), in the case when $c=0$, leading to some interesting results (see, e.g., the works [7, 10-14]). Moreover, it has been recently seen that, contrary to the classical case, models similar to the one taken into account in the present paper may display the existence of discontinuous traveling wave solutions. We refer the reader to the works $[15$, 16], in presence of more general operators, in relation with the so-called porous medium equation. Although we will not deal with discontinuous solutions, in Section 5 we shall refer to discontinuous steady states.

The plan of the paper is as follows. In Section 2, we will explicitly obtain the first order reduction for (4) (for a similar procedure, see for instance [17]) and state some elementary lemmas which will find application from there on. Section 3 is devoted to the study of the admissible speeds. Referring to the sign of $f(u)$ in $[0,1]$, we will mainly take into account the following three model cases: $f$ always positive ('type A'), $f$ positive in an open left neighborhood of 1 and 0 else ('type $\mathrm{B}$ '), $f$ positive in a left neighborhood of 1 and negative in the complementary open neighborhood of 0 ('type C'). This terminology was introduced in [18], but these shapes of the reaction term were already considered in [19]. We will see that the picture for type $C$ functions can be extended to a quite general setting. In Section 4 we will briefly discuss a different perspective on equation (4), interpreting it as a quasilinear version of a damped pendulum when $f(u)=B \sin u$. In this context, the critical speed assumes the meaning of a critical damping and we will be able to recover, with some differences, the usual picture already present in the classical case, in the case that an additional torque is applied to the pendulum as well. Finally, in Section 5 we will discuss the relationships between the appearance of discontinuous steady states (i.e., discontinuous solutions to (4) for $c=0$ ) and the nonexistence of right moving traveling waves, inspired by the work [9].

The figures appearing in the paper have been drawn using the open source software available at the web address [20]; we warn the reader that the numerical method used for the simulations, also in view of the presence of a singularity in the considered equation, is quite sensitive to the initial data and may display some problems for certain values of the parameters.

\section{Traveling waves: the first order model}

We are looking for monotone solutions to the boundary value problem

$$
\left\{\begin{array}{l}
\left(P\left(u^{\prime}\right)\right)^{\prime}-c u^{\prime}+f(u)=0, \\
u(-\infty)=0, \quad u(+\infty)=1,
\end{array}\right.
$$


where

$$
P(v)=\frac{v}{\sqrt{1+v^{2}}} .
$$

In view of the monotonicity of $u(t)$, we can write $t$ as a function of $u$ and change variables by setting $\phi(u)=P\left(u^{\prime}(t(u))\right)$ and $v(u)=P^{-1}(\phi(u))$, leading to the equation

$$
\frac{d}{d u} Q(v(u))-c v(u)+f(u)=0,
$$

where $Q(v)$ is the primitive of $v P^{\prime}(v)$ satisfying $Q(0)=0$. Explicitly,

$$
Q(v)=1-\frac{1}{\sqrt{1+v^{2}}},
$$

so that

$$
R=Q^{-1} \Rightarrow R(y)=\frac{\sqrt{y(2-y)}}{1-y}, \quad 0 \leq y<1 .
$$

Hence, we obtain the first order equation

$$
y^{\prime}=c R(y)-f(u)
$$

where the differentiation is done with respect to the independent variable $u$. Taking into account the boundary conditions, we thus want to study

$$
\left\{\begin{array}{l}
y^{\prime}=c \frac{\sqrt{y(2-y)}}{1-y}-f(u) \\
y(0)=0=y(1)
\end{array}\right.
$$

searching for the values of $c$ yielding a positive solution on ]0,1[ (the so-called admissible speeds); they will be obtained by shooting backward the solution to

$$
\left\{\begin{array}{l}
y^{\prime}=c \frac{\sqrt{y(2-y)}}{1-y}-f(u) \\
y(1)=0
\end{array}\right.
$$

and making it intersect, for suitable value(s) of $c$, with a solution to

$$
\left\{\begin{array}{l}
y^{\prime}=c \frac{\sqrt{y(2-y)}}{1-y}-f(u) \\
y(0)=0
\end{array}\right.
$$

Observe indeed that, in view of the fact that $R(y)=(\sqrt{y(2-y)}) /(1-y)$ is increasing in $[0,1[$, the solution to (8) is unique; for fixed $c$, we denote it by $y_{c, f}^{-}(u)$.

Different forms of the reaction term $f$ will be considered. However, we will always assume that

$$
\left\{\begin{array}{l}
f:[0,1] \rightarrow \mathbb{R} \text { is continuous, with } f(0)=0=f(1) ; \\
\text { there exists } u_{0} \in\left[0,1\left[\text { such that } f\left(u_{0}\right)=0 \text { and } f(u)>0 \text { in }\right] u_{0}, 1[\right.
\end{array}\right.
$$


(without the last requirement, no positive solutions to (8) could exist, as will be clear also from Lemmas 2.5 and 2.6 below). If $u_{0}=0$, then the positivity of $f$ yields the necessary condition $c>0$, as can be seen directly integrating the differential equation in (5). It will thus be natural to focus on positive speeds, as we will do henceforth.

Remark 2.1 We observe that the solutions to (5) are recovered, up to time translations, by solving the Cauchy problem

$$
\left\{\begin{array}{l}
u^{\prime}(t)=\frac{\sqrt{y(u(t))(2-y(u(t)))}}{1-y(u(t))}, \\
u(0)=\frac{1}{2},
\end{array}\right.
$$

similar to [21, Proposition 2.3].

Remark 2.2 The solution to the above Cauchy problem is defined in the whole real line and takes values in ]0,1[ provided that $f$ satisfies the conditions

(i) there exists $k>0$ such that $f(u) \leq k u$ for every $u \in[0,1]$,

(ii) there exists $l>0$ such that $f(u) \leq l(1-u)$ for every $u \in[0,1]$

(see $[21,22])$. The traveling wave profile is then a true heteroclinic connection from 0 to 1 , otherwise we may speak of heteroclinics that are degenerate (in the sense that they have a constant tail).

Thus problem (7) embodies (5). When we refer to admissible speeds, we have (7) in mind; anyway the existence of solutions to (7) in practice depends on condition (i) (see Proposition 3.2 below).

Remark 2.3 If one wished to search for decreasing solutions, namely $u^{\prime}(t(u))<0$, the model to be considered would be the same, but the variable $v$ in (6) would be negative. This would lead to the Cauchy problem

$$
\left\{\begin{array}{l}
y^{\prime}=-c \frac{\sqrt{y(2-y)}}{1-y}-f(u), \\
y(0)=0, \quad y(u) \geq 0 .
\end{array}\right.
$$

In this case, wishing to look at solutions which are decreasing in $t$, arriving at a certain point $u_{0}$ with 0 derivative, one should shoot forward from $u_{0}$.

\subsection{Preliminary lemmas}

In this subsection, we present some preliminary lemmas for the Cauchy problems (8) and (9). They mainly follow from elementary and well-known facts about subsolutions and supersolutions, but for the sake of simplicity we prefer to prove them explicitly in our particular case.

The first lemma concerns some general considerations about the solution $y_{c, f}^{-}(u)$, for $c$, $f$ fixed.

Lemma 2.4 Let $f$ satisfy (f). Then, for every $c>0$,

$$
y_{c, f}^{-}(u)<1 \text { for every } u \in[0,1]
$$


and

$$
\left.\left.y_{c, f}^{-}(u)>0 \quad \text { for every } u \in\right] u_{0}, 1\right]
$$

Proof We first prove (10). Assume by contradiction that there exists $\bar{u} \in[0,1[$ such that

$$
\limsup _{u \rightarrow \bar{u}^{+}} y_{c, f}^{-}(u)=1
$$

since $y_{c, f}^{-}$is continuous and $y_{c, f}^{-}(1)=0$, we can assume, without loss of generality, that

$$
y_{c, f}^{-}(u)<1 \quad \text { for } u>\bar{u} .
$$

Then, using the differential equation in (8), we would be able to construct a sequence $a_{n} \rightarrow \bar{u}$ with the contradictory properties $\left(y_{c, f}^{-}\right)^{\prime}\left(a_{n}\right) \leq 0$ and

$$
\lim _{n \rightarrow \infty}\left(y_{c, f}^{-}\right)^{\prime}\left(a_{n}\right)=+\infty
$$

We now observe that $z(u) \equiv 0$ is a lower solution for $(8)$ in $\left[u_{0}, 1\right]$, so that $y_{c_{f}}^{-}(u) \geq 0$ for every $u \in\left[u_{0}, 1\right]$. On the other hand, if there existed $\left.\left.u_{1} \in\right] u_{0}, 1\right]$ with $y_{c_{f} f}^{-}\left(u_{1}\right)=0$, then the differential equation in (8) would yield $\left(y_{c_{f}}^{-}\right)^{\prime}\left(u_{1}\right)<0$, implying that $y_{c_{f},}^{-}$is decreasing in a neighborhood of $u_{1}$, a contradiction. Hence, (11) is proved.

With the following two lemmas, we now take into account some monotonicity issues for $y_{c, f}^{-}(u)$ with respect to $c$ and $f$.

Lemma 2.5 Let $0<c_{1}<c_{2}$. Then, with the above notation, we have

$$
\left.y_{c_{1}, f}^{-}(u) \geq y_{c_{2}, f}^{-}(u) \quad \text { for every } u \in\right] 0,1[
$$

and

$$
\left.y_{c_{1}, f}^{-}(u)>y_{c_{2}, f}^{-}(u) \quad \text { for every } u \in\right] u_{0}, 1[\text {. }
$$

If $y_{c_{2}, f}^{-}\left(u_{0}\right)>0$, in particular, then $y_{c_{1}, f}^{-}\left(u_{0}\right)>y_{c_{2}, f}^{-}\left(u_{0}\right)$.

Proof Assume first that there exists a point $\left.u^{*} \in\right] 0,1\left[\right.$ such that $y_{c_{1}, f}^{-}\left(u^{*}\right)<y_{c_{2}, f}^{-}\left(u^{*}\right)$; by continuity, the inequality can be extended to some maximal interval $I=\left[u^{*}, u^{+}[\right.$, being $y_{c_{1}, f}^{-}\left(u^{+}\right)=y_{c_{2}, f}^{-}\left(u^{+}\right)$. By the strict monotonicity of $R(y)$ we have, for $u \in I$,

$$
0 \leq y_{c_{2}, f}^{-}(u)-y_{c_{2}, f}^{-}\left(u^{+}\right)+y_{c_{1}, f}^{-}\left(u^{+}\right)-y_{c_{1}, f}^{-}(u) \leq c_{2} \int_{u}^{u^{+}} R\left(y_{c_{1}, f}^{-}(\tau)\right)-R\left(y_{c_{2}, f}^{-}(\tau)\right) d \tau<0,
$$

a contradiction. Thus, $y_{c_{2}, f}^{-}(u) \leq y_{c_{1}, f}^{-}(u)$ for every $\left.u \in\right] 0,1[$.

On the other hand, if there existed $\left.u^{\prime} \in\right] u_{0}, 1\left[\right.$ such that $0<y_{c_{2}, f}^{-}\left(u^{\prime}\right)=y_{c_{1}, f}^{-}\left(u^{\prime}\right)$ (recall that, in $] u_{0}, 1\left[\right.$, the solution to (8) is strictly positive), then, since $c_{1}<c_{2}$, using the differential equation in (8) we would have $\left(y_{c_{1},}^{-}\right)^{\prime}(u)-\left(y_{c_{2}, f}^{-}\right)^{\prime}(u)<0$ in a neighborhood of $u^{\prime}$, which is impossible because $y_{c_{1}, f}^{-}(u)-y_{c_{2}, f}^{-}(u) \geq 0$ for every $\left.u \in\right] u_{0}, 1[$. This gives the conclusion for the case $y_{c_{2}, f}^{-}\left(u_{0}\right)>0$, as well. 
If $u_{0}=0$, this yields in particular a strict monotonicity of the solutions, with respect to the constant $c$, along the open interval ]0,1[.

Lemma 2.6 Let $c \geq 0$ be fixed and $f_{1}, f_{2}$ satisfy $(\mathrm{f}), f_{1} \geq f_{2}$ in $[\hat{u}, 1]$ with $f_{1}(u)>f_{2}(u)$ for every $u \in\left[\hat{u}, \tilde{u}[\right.$ (where $0<\hat{u}<\tilde{u} \leq 1)$. Then, $y_{c, f_{1}}^{-}(u) \geq y_{c, f_{2}}^{-}(u) \forall u \in[\hat{u}, 1]$ and

$$
y_{c, f_{1}}^{-}(u)>y_{c, f_{2}}^{-}(u) \text { for every } u \in[\hat{u}, \tilde{u}[\text {. }
$$

Proof The proof is similar to the one of the previous lemma. Indeed, if there existed a point $u^{*} \in\left[\hat{u}, 1\left[\right.\right.$ such that $y_{c, f_{1}}^{-}\left(u^{*}\right)<y_{c, f_{2}}^{-}\left(u^{*}\right)$, one could find again an interval $I=\left[u^{*}, u^{+}[\right.$ such that $y_{c, f_{1}}^{-}\left(u^{+}\right)=y_{c, f_{2}}^{-}\left(u^{+}\right)$and $y_{c, f_{1}}^{-}<y_{c, f_{2}}^{-}$on $I$. By the strict monotonicity of $R(y)$ it would then be, for $u \in I$,

$$
\begin{aligned}
0 & \leq y_{c, f_{2}}^{-}(u)-y_{c, f_{2}}^{-}\left(u^{+}\right)+y_{c, f_{1}}^{-}\left(u^{+}\right)-y_{c, f_{1}}^{-}(u) \\
& =\int_{u}^{u^{+}}\left(f_{2}(u)-f_{1}(u)\right) d u+c \int_{u}^{u^{+}} R\left(y_{c, f_{1}}^{-}(\tau)\right)-R\left(y_{c, f_{2}}^{-}(\tau)\right) d \tau<0,
\end{aligned}
$$

yielding a contradiction. Hence, $y_{c, f_{1}}^{-}(u) \geq y_{c, f_{2}}^{-}(u)$ for every $u \in[\hat{u}, 1[$.

On the other hand, if there existed $v \in\left[\hat{u}, \tilde{u}\left[\right.\right.$ such that $y_{c, f_{1}}^{-}(v)=y_{c, f_{2}}^{-}(v)$, we would have $\left(y_{c, f_{1}}^{-}\right)^{\prime}(v)<\left(y_{c, f_{2}}^{-}\right)^{\prime}(v)$ which is impossible.

We finally analyze the behavior of the maximum of $y_{c, f}^{-}(u)$, in dependence of a small $c$.

Lemma 2.7 The limit $\mathcal{M}:=\lim _{c \rightarrow 0^{+}} \max _{u \in\left[u_{0}, 1\right]} y_{c, f}^{-}(u)$ exists. Moreover, we have

$$
\mathcal{M}=\min \left\{\int_{u_{0}}^{1} f(u) d u, 1\right\}
$$

Proof Assume first that $\int_{u_{0}}^{1} f(u) d u \geq 1$ and suppose, by contradiction, that there exist $\delta>0$ and a decreasing sequence $c_{n} \rightarrow 0^{+}$such that $\max _{u \in\left[u_{0}, 1\right]} y_{c_{n} f}^{-}(u) \leq 1-\delta$ for every $n$ (we know that this sequence has a limit since it is monotone in view of the previous lemmas; moreover, such a limit is less than or equal to 1 in view of (10)). Let $t_{n} \in\left[u_{0}, 1\right]$ be the point where $y_{c_{n} f}^{-}$reaches its maximum and observe that $t_{n}$ is bounded away from 1 , since $y_{c_{n}, f}^{-}(1)=0$ for every $n$ and $y_{0, f}^{-}$reaches its maximum in $u_{0}$ (recall that $c_{n} \rightarrow 0^{+}$). Moreover, $t_{n} \neq u_{0}$ for every $n$, since either $y_{c_{n}, f}^{-}\left(u_{0}\right)=0$ or $\left(y_{c_{n}, f}^{-}\right)^{\prime}\left(u_{0}\right)>0$. Since $\left(y_{c_{n}, f}^{-}\right)^{\prime}\left(t_{n}\right)=0$, we have

$$
f\left(t_{n}\right)=c_{n} \frac{\sqrt{y_{c_{n}, f}^{-}\left(t_{n}\right)\left(2-y_{c_{n}, f}^{-}\left(t_{n}\right)\right)}}{1-y_{c_{n}, f}^{-}\left(t_{n}\right)}
$$

from which, as $t_{n}$ converges, up to subsequences, to a certain $t_{0}$, we deduce $f\left(t_{0}\right)=0$. It follows that $t_{0}=u_{0}$, and in view of

$$
\int_{t_{n}}^{1}\left[f(u)-c_{n} \frac{\sqrt{y_{c_{n}, f}^{-}(u)\left(2-y_{c_{n}, f}^{-}(u)\right)}}{1-y_{c_{n}, f}^{-}(u)}\right] d u=y_{c_{n}, f}^{-}\left(t_{n}\right) \leq 1-\delta
$$

we infer, for $n \rightarrow+\infty$, that $\int_{u_{0}}^{1} f(u) d u \leq 1-\delta$, contrary to the assumption. 
In the case when $\int_{u_{0}}^{1} f(u) d u<1$, we argue analogously to obtain that $\mathcal{M} \geq \int_{u_{0}}^{1} f(u) d u$, assuming at the beginning that $y_{c_{n} f}^{-}\left(t_{n}\right) \leq \int_{u_{0}}^{1} f(u) d u-\delta$. For the reverse inequality, we first observe that $\mathcal{M}<1$ since $y_{c_{n} f}^{-}\left(t_{n}\right)<\max _{u \in\left[u_{0}, 1\right]} y_{0, f}^{-}(u)<1$ (in view of Lemma 2.5) and then reason as in (12).

The last lemma concerns the solutions shot forward from 0 .

Lemma 2.8 Assume that there exists $\left.\left.u_{1} \in\right] 0,1\right]$ with $f(u)>0$ for $\left.u \in\right] 0, u_{1}\left[\right.$ and $f\left(u_{1}\right)=0$. Let $y_{c, f}^{+}(u)$ be a (forward) solution to (9). Then,

$$
\max _{u \in\left[0, u_{1}\right]} y_{c, f}^{+}(u) \rightarrow 0 \quad \text { for } c \rightarrow 0^{+}
$$

(where it is understood that $y_{c, f}^{+}$is replaced with 0 if it takes negative values, in order for the equation in (9) to make sense).

Proof By contradiction, if there existed $\delta>0$ and, given $c_{n} \rightarrow 0^{+}$, an interval $\left[t_{n}^{-}, t_{n}^{+}\right] \subset$ $\left[0, u_{1}\right]$ such that $y_{c_{n}, f}^{+}\left(t_{n}^{-}\right)=\delta / 2, y_{c_{n}, f}^{+}\left(t_{n}^{+}\right)=\delta$, and $\delta / 2<y_{c_{n}, f}^{+}(u)<\delta$ for every $\left.u \in\right] t_{n}^{-}, t_{n}^{+}[$, one would have, for a suitable $H>0$,

$$
\begin{aligned}
\delta / 2 & =y_{c_{n}, f}^{+}\left(t_{n}^{+}\right)-y_{c_{n}, f}^{+}\left(t_{n}^{-}\right)=c_{n} \int_{t_{n}^{-}}^{t_{n}^{+}} \frac{\sqrt{y_{c_{n}, f}(u)\left(2-y_{c_{n}, f}(u)\right)}}{1-y_{c_{n}, f}(u)} d u-\int_{t_{n}^{-}}^{t_{n}^{+}} f(u) d u \\
& \leq c_{n} \frac{\sqrt{\delta(2-\delta / 2)}}{1-\delta}\left(t_{n}^{+}-t_{n}^{-}\right)-H,
\end{aligned}
$$

which gives a contradiction for $n \rightarrow+\infty$.

\section{Traveling waves: the admissible speeds}

We now turn to the search for solutions to (7), under some classical assumptions on $f$.

\section{1 $f$ of type A}

As a first case, we will assume that the reaction term $f$ is of type A. Precisely, we define

$$
\mathcal{A}=\{f \in C([0,1]) \mid f(0)=f(1)=0 \text { and } f(u)>0 \text { for every } u \in] 0,1[\} .
$$

In this case, Lemmas $2.4,2.5$, and 2.7 hold with $u_{0}=0$, so that, in particular, $y_{c, f}^{-}$is positive in $] 0,1[$.

Under some mild assumptions on $f$, we first give a lower bound for the admissible speeds.

Lemma 3.1 Let $k:=f^{\prime}(0)$ exist and let $y(u)$ be a solution to (7), with $y(u)>0$ in a right neighborhood of 0 . Then, $c \geq 2 \sqrt{f^{\prime}(0)}$.

Proof The proof is the same as the one in [22], but we recall it for the reader's convenience. Let $y(u)$ be positive for $u \in] 0, \hat{u}\left[\right.$; then, $(\sqrt{y(u)})^{\prime}$ is defined, and

$$
l:=\limsup _{u \rightarrow 0^{+}}(\sqrt{y(u)})^{\prime} \geq 0
$$


since $y(0)=0$ and $y(u)>0$ in $] 0, \hat{u}[$. However,

$$
\begin{aligned}
(\sqrt{y(u)})^{\prime} & =\frac{1}{2 \sqrt{y(u)}} y^{\prime}(u)=\frac{1}{2 \sqrt{y(u)}}\left[c \frac{\sqrt{y(u)(2-y(u))}}{1-y(u)}-f(u)\right] \\
& =\frac{1}{2}\left[c \frac{\sqrt{(2-y(u))}}{1-y(u)}-\frac{f(u)}{u} \frac{u}{\sqrt{y(u)}}\right]
\end{aligned}
$$

and, taking the upper limit for $u \rightarrow 0^{+}$at both sides, having $y(0)=0$, we obtain

$$
l \leq \frac{1}{2}\left[\sqrt{2} c-\liminf _{u \rightarrow 0^{+}} \frac{f(u)}{u} \frac{u}{\sqrt{y(u)}}\right] .
$$

We can assume $f^{\prime}(0)>0$, otherwise the statement is trivially satisfied, as $c>0$. It follows that

$$
l \leq \frac{1}{2}\left[\sqrt{2} c-k \frac{1}{l}\right]
$$

whence $l>0$ and

$$
2 l^{2}-\sqrt{2} c l+k \leq 0
$$

implying the desired bound on $c$.

From Lemmas 2.7 and 2.8 , if $c$ is sufficiently small so that

$$
\max _{u \in[0,1]} y_{c, f}^{+}(u)<\max _{u \in[0,1]} y_{c, f}^{-}(u)
$$

where $y_{c, f}^{+}$is any solution to (9), it follows that $y_{c, f}^{-}(0)>0$ and no solutions to (7) can appear. Referring to the lemmas in Section 2.1, this simple observation allows one to realize that, in the case when $u_{0}>0$, there exists a maximal $c^{*}>0$ such that, for every $0<c<c^{*}, y_{c, f}^{-}\left(u_{0}\right)>$ 0 (as we will see, $c^{*}$ takes the name of critical speed). As a consequence, if in Lemma 2.5 we have $c_{1}<c_{2}$ and $c_{1}$ is sufficiently small, $y_{c_{1} f}^{-}\left(u_{0}\right)>y_{c_{2} f}^{-}\left(u_{0}\right)$.

Given a reaction term $f$ belonging to a quite large subset of $\mathcal{A}$, the following proposition relates the corresponding set of the admissible speeds to some bound on $f$.

Proposition 3.2 Let $f \in \mathcal{A}$ and assume that there exists $M>0$ such that the following estimate holds:

$$
f(u) \leq \frac{M u}{\sqrt{1-\min \{M, 1\} u^{2}}}
$$

for every $u \in[0,1]$. Then, for every

$$
c \in[2 \sqrt{M},+\infty[
$$


Proof Let us fix $c \geq 2 \sqrt{M}$ and consider the (backward) Cauchy problem (8), which has the unique global, positive solution $y_{c, f}^{-}(u)$ in $] 0,1\left[\right.$. If $y_{c, f}^{-}(0)=0$ we are done, so we assume $0<y_{c, f}^{-}(0)(<1)$.

Let us consider the (forward) Cauchy problem (9); we construct a positive lower solution by using the solutions to

$$
\left\{\begin{array}{l}
y^{\prime}=\beta \frac{\sqrt{y(2-y)}}{1-y} \\
y(0)=0
\end{array}\right.
$$

in dependence of $\beta>0$. Problem (13) has the solution

$$
z(u)=1-\sqrt{1-\beta^{2} u^{2}}
$$

for $u \in\left[0, u_{\beta}\right.$ [, where $u_{\beta}=1 / \beta$; we thus want to choose $\beta$ in such a way that $z(u)$ solves the differential inequality

$$
w^{\prime} \leq c R(w)-f(u)
$$

in a right neighborhood of $u=0$. Computing explicitly $R(z(u))$, we obtain

$$
R(z(u))=\frac{\sqrt{\left(1-\sqrt{1-\beta^{2} u^{2}}\right)\left(1+\sqrt{1-\beta^{2} u^{2}}\right)}}{\sqrt{1-\beta^{2} u^{2}}}=\frac{\beta u}{\sqrt{1-\beta^{2} u^{2}}},
$$

so that $z(u)$ will solve

$$
w^{\prime} \leq c R(w)-\frac{M u}{\sqrt{1-\beta^{2} u^{2}}}
$$

if and only if

$$
\frac{\beta^{2} u}{\sqrt{1-\beta^{2} u^{2}}} \leq \frac{\beta c u}{\sqrt{1-\beta^{2} u^{2}}}-\frac{M u}{\sqrt{1-\beta^{2} u^{2}}} .
$$

It follows that $\beta$ must solve the inequality

$$
\beta^{2}-\beta c+M \leq 0
$$

which, in view of the choice of $c$ (namely $c \geq 2 \sqrt{M}$ ), has at least a solution. We choose

$$
\beta=\frac{c+\sqrt{c^{2}-4 M}}{2}
$$

and observe that $c \geq 2 \sqrt{M}$ implies that

$$
\beta \geq \sqrt{M} \geq \sqrt{\min \{M, 1\}} .
$$


For every $u \in] 0, u_{\beta}[$, we thus have

$$
\frac{M u}{\sqrt{1-\min \{M, 1\} u^{2}}} \leq \frac{M u}{\sqrt{1-\beta^{2} u^{2}}}
$$

so that

$$
z^{\prime} \leq c R(z)-\frac{M u}{\sqrt{1-\beta^{2} u^{2}}} \leq c R(z)-f(u), \quad z(0)=0
$$

for $u \in] 0, u_{\beta}[$. This means that $z(u)$ is a positive lower solution to (9) in the interval $] 0, u_{\beta}[$. As a consequence, there exists a solution $y_{c, f}^{+}(u)$ to $(9)$ which satisfies

$$
\left.y_{c, f}^{+}(u) \geq z(u)>0 \quad \text { for every } u \in\right] 0, u_{\beta}[
$$

Now consider two cases.

If $y_{c, f}^{+}(u)$ blows up at a finite time $\left.u_{\infty} \in\right] 0,1[$, then there exists $\hat{u} \in] 0, u_{\infty}[$ such that $y_{c, f}^{+}(\hat{u})>\max _{u \in[0,1]} y_{c, f}^{-}(u)$. Since $y_{c, f}^{+}$is of class $C^{1}$ and the graph of $y_{c, f}^{-}(u)$ disconnects the square $[0,1]^{2}$ in the $(u, y)$-plane, this implies that $y_{c, f}^{+}(\bar{u})=y_{c, f}^{-}(\bar{u})$ for some $\left.\bar{u} \in\right] 0, \hat{u}[$, which is impossible by uniqueness.

If $y_{c, f}^{+}(u)$ is globally defined in $[0,1]$, the same happens for $z(u)$; then, recalling $(14), y_{c, f}^{+}(u)$ is always strictly positive in $] 0,1\left[\right.$. Thus, either $y_{c, f}^{+}(1)=0$ and we are done, or we can argue similarly as in the previous case to deduce that $y_{c, f}^{+}(\bar{u})=y_{c, f}^{-}(\bar{u})$ at some point $\left.\bar{u} \in\right] 0,1[$, which is impossible by uniqueness. This concludes the proof.

It is worth observing that, differently from the classical and the Minkowski case, in our setting the family of functions giving the bound on $f$ may be unbounded on $[0,1]$. We explicitly remark, moreover, that in order to perform the previous proof it is essential that $y_{c, f}^{-}(u)$ disconnects the square $[0,1]^{2}$ in the $(u, y)$-plane, thus forming a kind of barrier which has to be crossed by the forward solution $y_{c, f}^{+}(u)$ (also this point is different from the classical case).

As an example, given $M>1$, if $f \in \mathcal{A}$ is given by

$$
f(u)=\frac{M u}{\sqrt{1-u^{2}}}
$$

in a neighborhood of $u=0$, then every $c \geq 2 \sqrt{f^{\prime}(0)}$ is admissible for our problem (since $f^{\prime}(0)=M$ in this case) .

We now focus on some characterizations of the so-called critical speed - i.e., the value $c^{*}$ such that every $c \geq c^{*}$ is admissible - for functions of type A. In the previous example (15), for instance, we have $c^{*}=2 \sqrt{M}$.

We will proceed sketchily, since the method used is the same as in [21]. We first observe that

$$
\lim _{y \rightarrow 0^{+}} \frac{R(y)}{\sqrt{y}}=\sqrt{2},
$$


so that, setting $E(y)=\int_{0}^{y} \frac{1}{R(z)} d z=\sqrt{y(2-y)}$, we have

$$
\lim _{y \rightarrow 0^{+}} \frac{E(y)}{R(y)}=1 \text {. }
$$

Moreover, since by (16) the solutions to (9) satisfy, in a neighborhood of $u=0$,

$$
y^{\prime} \leq c(\sqrt{2}+\epsilon) \sqrt{y}
$$

we deduce that $y(u) \leq k u^{2}$ in a neighborhood of 0 , where $k>0$ is a suitable constant. These two facts are sufficient to prove the validity of the following proposition, just as in [21, Proposition 3.4].

Proposition 3.3 Let $f$ satisfy (f) and let $f^{\prime}(0)$ exist. If $y(u)$ is a solution to the differential equation in (9) such that $y(0)=0$ and $y(u)>0$ in a right neighborhood of 0 , then the quantity

$$
E(y)^{\prime}(0)=\left.\frac{d}{d u} E(y(u))\right|_{u=0}
$$

exists and is a root of the equation $x^{2}-c x+f^{\prime}(0)=0$.

To characterize the critical speed, we now need the following analog of [21, Lemma 4.1].

Proposition 3.4 Fix $\epsilon>0$ small. Let $\eta>0$ be sufficiently small and $0<A<B<1 / \eta, 0 \leq$ $a<b, 2 \sqrt{b}<c_{1}<c_{2}<\frac{\sqrt{2} A}{1+\epsilon}$ be constants such that

$$
\left.\left.a \leq \frac{f(u)}{u} \leq b \quad \text { for every } u \in\right] 0, \eta\right]
$$

and

$$
2 A^{2}-c \sqrt{2} A+b<0<2 B^{2}-c \sqrt{2} B+a \text { for every } c \in\left[c_{1}, c_{2}\right]
$$

Then, decreasing $\eta$ if necessary, for every $c \in\left[c_{1}, c_{2}\right]$ there exists a unique solution $y(u)$ to (9) such that $A^{2} u^{2} \leq y(u) \leq B^{2} u^{2}$ for every $u \in[0, \eta]$.

The only difference, making a comparison with [21, Lemma 4.1], is that we have to pose the further requirement $B<1 / \eta$ to ensure that $y(u)$ stays bounded away from the barrier $y=1$. The proof exploits the Banach-Caccioppoli theorem applied on the space

$$
\mathcal{E}=\left\{y \in C([0,1]) \mid A^{2} u^{2} \leq y(u) \leq B^{2} u^{2} \text { for every } u \in[0, \eta]\right\}
$$

endowed with the norm $\|y\|=\sup _{0<t \leq \eta} \frac{y(t)}{t}$. Indeed, thanks to (16), the operator $T$ defined by

$$
T y(t)=c \int_{0}^{t} R(y(u)) d u-\int_{0}^{t} f(u) d u
$$


maps $\mathcal{E}$ into itself and, shrinking $\eta$ if necessary (in dependence of $\epsilon$ ), for every $u \in[0, \eta]$ and $y, z \in \mathcal{E}$ the following holds:

$$
|T(y(u))-T(z(u))| \leq \frac{c \sqrt{2}}{2 A}(1+\epsilon) u\|y-z\|
$$

implying that $T$ is a contraction if $c<\frac{\sqrt{2} A}{1+\epsilon}$.

At this point, denoting by

$$
\lambda_{-}(c) \leq \lambda_{+}(c)
$$

the roots of the equation $x^{2}-c x+f^{\prime}(0)=0$, we have the following.

Proposition 3.5 Under the previous assumptions on $f(u)$, let $c$ be an admissible speed for (7) and $y(u)$ be the corresponding solution. We have:

- if $c=c^{*}$, then

$$
E(y)^{\prime}(0)=\lambda^{+}(c)
$$

- if $c>c^{*}$, then

$$
E(y)^{\prime}(0)=\lambda^{-}(c)
$$

The proof goes as the one for [21, Proposition 4.2], with minor changes.

We finally give some examples of exact solutions in the case when $f \in \mathcal{A}$ has some special form (taking inspiration from [21, 22]).

(1) By analogy with the classical Fisher equation and [21, Example 1], let

$$
f(u)=\frac{u(1-u)}{\sqrt{1-\beta u^{2}(1-\sqrt{u})^{2}}}
$$

with $\beta>0$. An explicit computation shows that

$$
y(u)=1-\sqrt{1-\beta u^{2}(1-\sqrt{u})^{2}}
$$

is a solution for $\beta=2 / 3$ and $c=5 / \sqrt{6}$. In this case, $f^{\prime}(0)=1$; since

$$
f(u) \leq \frac{u}{\sqrt{1-u^{2}}}
$$

in view of Lemma 3.1 and Proposition 3.2 we conclude that $c^{*}=2$. Hence, the solution found does not correspond to a critical speed, as it is possible to see also using Proposition 3.5:

$$
E(y)^{\prime}(0)=\left.\frac{d}{d u} \sqrt{\beta u^{2}(1-\sqrt{u})^{2}}\right|_{u=0}=\sqrt{\beta}=\sqrt{2 / 3}=\lambda_{-}(5 / \sqrt{6}) .
$$


(2) By analogy with the Zeldovich equation, if

$$
f(u)=\frac{u^{2}(1-u)}{\sqrt{1-\beta\left(u-u^{2}\right)^{2}}}
$$

we find a solution of the form

$$
y(u)=1-\sqrt{1-\beta\left(u-u^{2}\right)^{2}}
$$

for $\beta=1 / 2$ and $c=1 / \sqrt{2}$. In this case, $f^{\prime}(0)=0$, and since

$$
E(y)^{\prime}(0)=\left.\frac{d}{d u} \sqrt{\beta}\left(u-u^{2}\right)\right|_{u=0}=\sqrt{\beta}=1 / \sqrt{2}=\lambda_{+}(1 / \sqrt{2})
$$

we conclude that $c=1 / \sqrt{2}$ is the critical speed. Notice that, in this case, the bound

$$
f(u) \leq \frac{\beta u}{\sqrt{1-\beta u^{2}}}
$$

for every $u \in[0,1]$ would provide only a rougher estimate of $c^{*}$ via Proposition 3.2.

\section{$3.2 f$ of type B}

We now take into account other forms of the reaction term $f$; in this subsection, in particular, we will assume that $f(u)$ is of type B in $[0,1]$. For the sake of simplicity, we define

$$
\mathcal{B}=\left\{\begin{array}{l|l}
f \in C([0,1]) & \begin{array}{l}
f(0)=f(1)=0 \text { and there exists } \theta \in] 0,1[\text { s.t. } \\
f(u)=0 \text { for } u \in[0, \theta], f(u)>0 \text { for } u \in] \theta, 1[
\end{array}
\end{array} .\right.
$$

In this setting, we have the following result, similar to the ones in the classical and in the Minkowski case (see also [23]).

Proposition 3.6 Let $f \in \mathcal{B}$. Then there exists $0<c^{*}<1 / \theta$ such that (7) has a solution if and only if $c=c^{*}$.

Proof In the interval $[0, \theta]$, the positive solution to (9) is unique; we denote it by $y_{c, f}^{+}$and observe that its explicit expression is given by $y_{c, f}^{+}(u)=1-\sqrt{1-c^{2} u^{2}}$, so that

$$
y_{c, f}^{+}(\theta)=1-\sqrt{1-c^{2} \theta^{2}}
$$

On the other hand, the unique solution to

$$
\left\{\begin{array}{l}
y^{\prime}=c \frac{\sqrt{y(2-y)}}{1-y} \\
y(1)=0
\end{array}\right.
$$

given explicitly by $\bar{y}(u)=1-\sqrt{1-c^{2}(u-1)^{2}}$, is a subsolution for (8) in $] \theta, 1[$, so that, for $c>0$,

$$
y_{c, f}^{-}(\theta) \geq \bar{y}(\theta)=1-\sqrt{1-c^{2}(\theta-1)^{2}}>0 .
$$


To obtain a solution to our original problem, we need $y_{c, f}^{+}(\theta)=y_{c, f}^{-}(\theta)$. However, $y_{c, f}^{+}(\theta)$ and $y_{c, f}^{-}(\theta)$ depend continuously on $c$ (the first in view of its explicit expression, the second by the standard theory of ODEs); moreover, if $c$ increases from 0 to $1 / \theta$, then $y_{c, f}^{+}(\theta)$ strictly increases from 0 to 1 (spanning all the possible values strictly included), while $y_{c, f}^{-}(\theta)$ is nonincreasing, as given by Lemma 2.5. This is sufficient to conclude the existence of at least one admissible speed $c^{*}$ (for which $y_{c^{*}, f}^{+}(\theta)=y_{c^{*}, f}^{-}(\theta)$ ), in view of the intermediate value theorem.

The fact that $c^{*}$ is unique follows easily: taking $c^{\prime} \neq c$, in view of the explicit expression of $y_{c, f}^{+}(u)$ and Lemma 2.5, we have either

$$
y_{c^{\prime}, f}^{+}(\theta)<y_{c^{*}, f}^{+}(\theta)=y_{c^{*}, f}^{-}(\theta) \leq y_{c^{\prime}, f}^{-}(\theta)
$$

(if $c^{\prime}<c^{*}$ ) or

$$
y_{c^{\prime}, f}^{+}(\theta)>y_{c^{*}, f}^{+}(\theta)=y_{c^{*}, f}^{-}(\theta) \geq y_{c^{\prime}, f}^{-}(\theta)
$$

(if $c^{\prime}>c^{*}$ ), so that $y_{c^{\prime}, f}^{-}(\theta) \neq y_{c^{\prime}, f}^{+}(\theta)$.

Similar to the classical case, the lower bound for the admissible speeds of a function $f$ of type A can be reconstructed starting from a sequence of increasing approximations of type B. Precisely, we have the following proposition.

Proposition 3.7 Let $f \in \mathcal{A}$ with finite critical speed $c^{*}$ and let $f_{n} \in \mathcal{B}$ such that $f_{n} \nearrow f$ pointwise. Then,

$$
c_{n}:=c^{*}\left(f_{n}\right) \nearrow c^{*}(f)
$$

Before going into the details of the proof, let us explicitly state that, if $\theta_{n}>0$ is the greatest zero of $f_{n}$ on $\left[0,1\left[\right.\right.$, we assume that $\theta_{n}$ is strictly decreasing and $f_{n} \leq f_{n+1}$.

Proof The proof is similar to the one for the classical case. We divide it in four steps. To fix the notation, denote by $y_{c, f}, y_{c, f}^{-}, y_{c, f}^{+}$the solutions to (7), (8), (9), respectively; for briefness, we write $y_{n}, y_{n}^{+}, y_{n}^{-}$to denote $y_{c_{n} f_{n}}, y_{c_{n}, f_{n}}^{+}, y_{c_{n} f_{n}}^{-}$.

Step 1: $c^{*}\left(f_{n}\right)$ is increasing.

For every $n$, we have

$$
y_{n}^{\prime}=\frac{c_{n} \sqrt{y_{n}\left(2-y_{n}\right)}}{1-y_{n}}-f_{n} \geq \frac{c_{n} \sqrt{y_{n}\left(2-y_{n}\right)}}{1-y_{n}}-f_{n+1} .
$$

Hence, since $f_{n}(u)<f_{n+1}(u)$ in some interval $\left[\theta_{n}, \theta_{n}+\delta_{n}[\right.$, Lemma 2.6 ensures that

$$
y_{c_{n} f_{n+1}}^{+}\left(\theta_{n}\right) \leq y_{n}^{+}\left(\theta_{n}\right)=y_{n}^{-}\left(\theta_{n}\right)<y_{c_{n}, f_{n+1}}^{-}\left(\theta_{n}\right) .
$$

Thus, in view of Lemma 2.5 and the previous remarks, we have $c_{n+1}>c_{n}$. 
Step 2: for every $n, c^{*}\left(f_{n}\right) \leq c^{*}(f)$.

Reasoning as in Step 1, the fact that, for every $n, f(u)>f_{n}(u)$ for every $\left.u \in\right] 0, \theta_{n}[$ implies that, for every $n$,

$$
y_{c_{n} f}^{+}\left(\theta_{n}\right) \leq y_{n}^{+}\left(\theta_{n}\right)=y_{n}^{-}\left(\theta_{n}\right)<y_{c_{n} f}^{-}\left(\theta_{n}\right)
$$

so that $c_{n}$ cannot be admissible for $f$.

From Step 1 and Step 2, it follows that $c_{n}$ converges.

Step 3: there exists $0<H<1$ such that $\left\|y_{n}\right\|_{\infty} \leq H$ for every $n$.

Observing that $y_{n}(u)<1$ for every $t$, assume by contradiction that there exists $\left(u_{n}\right)_{n}$, with $u_{n} \in[0,1]$, such that

$$
y_{n}\left(u_{n}\right)=\left\|y_{n}\right\|_{\infty} \rightarrow 1
$$

It follows that $y_{n}^{\prime}\left(u_{n}\right)=0$, so that

$$
\frac{c_{n} \sqrt{y_{n}\left(u_{n}\right)\left(2-y_{n}\left(u_{n}\right)\right)}}{1-y_{n}\left(u_{n}\right)}=f_{n}\left(u_{n}\right) .
$$

The contradiction follows from the fact that the left-hand side goes to $+\infty$, since $c_{n}>0$ is increasing, while the right-hand side is bounded.

Step 4: $c^{*}\left(f_{n}\right) \nearrow c^{*}(f)$.

By contradiction, assume that there exists $\hat{c}<c^{*}(f)$ such that $c^{*}\left(f_{n}\right) \rightarrow \hat{c}$. From

$$
y_{n}^{\prime}=\frac{c_{n} \sqrt{y_{n}\left(2-y_{n}\right)}}{1-y_{n}}-f_{n}, \quad y_{n}(0)=0=y_{n}(1)
$$

and Step 3, we deduce that $y_{n}$ is a bounded sequence in $C^{1}([0,1])$. By the Arzelà-Ascoli theorem, there exists $y \in C([0,1])$ such that $y_{n} \rightarrow y$ uniformly in $[0,1]$. Passing to the limit in (17), we get

$$
y^{\prime}=\frac{\hat{c} \sqrt{y(2-y)}}{1-y}-f, \quad y(0)=0=y(1)
$$

so that $\hat{c} \geq c^{*}(f)$.

\section{3 $f$ of type C}

Another typical form of the reaction term, besides the ones already presented, is the socalled type C. Explicitly, we define

$$
\mathcal{C}=\left\{\begin{array}{l|l}
f \in C([0,1]) & \begin{array}{l}
f(0)=f(1)=0 \text { and there exists } \theta \in] 0,1[\text { s.t. } \\
f(u)<0 \text { for } u \in] 0, \theta[, f(u)>0 \text { for } u \in] \theta, 1[
\end{array}
\end{array}\right\} .
$$

In this case, the solution $y_{c, f}^{+}$shot from the left is unique, as can be proved, with minor changes, in the same way as [24, Theorem 3.9]. Therefore, we have the following counterpart of Lemma 2.5. 
Lemma 3.8 Let $0<c_{1}<c_{2}$. Then, the following holds:

$$
\left.y_{c_{2}, f}^{+}(u) \geq y_{c_{1}, f}^{+}(u) \quad \text { for every } u \in\right] 0,1\left[\text { s.t. } y_{c_{2}, f}^{+}(u)<1\right.
$$

and

$$
\left.y_{c_{2}, f}^{+}(u)>y_{c_{1}, f}^{+}(u) \quad \text { for every } u \in\right] 0, \theta\left[\text { s.t. } y_{c_{2}, f}^{+}(u)<1\right. \text {. }
$$

If $0<y_{c_{1}, f}^{+}(\theta)<1$, in particular, then $y_{c_{2}, f}^{+}(\theta)>y_{c_{1}, f}^{+}(\theta)$.

The proof is analogous to the one of Lemma 2.5, and for this reason we will omit it.

In the classical case, the behavior of functions of type $B$ or type $C$ with respect to the admissible speeds does not change. We are now going to see that, for the curvature case, the situation is somehow different, due to the fact that the 'barrier' $\{y=1\}$ has to be avoided in order to obtain a classical solution to our problem.

Proposition 3.9 Let $f \in \mathcal{C}$. Then there exists a positive admissible speed for $f$ if and only if the two following conditions simultaneously hold:

$$
\int_{0}^{1} f(u) d u>0
$$

and

$$
\int_{0}^{1} f^{-}(u) d u<1
$$

where $f^{-}(t)=\max \{-f(t), 0\}$. If this is true, the admissible speed is unique.

Proof

(1) We first show that, if (19) is violated, then every solution shot from the left blows up at a finite time. This is an easy consequence of the fact that, for every $c \geq 0$,

$$
y^{\prime}=\frac{c \sqrt{y(2-y)}}{1-y}-f(u) \geq-f(u)
$$

so that

$$
z(u)=\int_{0}^{u}-f(s) d s
$$

is a subsolution from the left. Lemma 3.8 now ensures that

$$
\left.y_{c, f}^{+}(u)>z(u) \quad \text { for every } u \in\right] 0, \theta[\text { s.t. } z(u)<1 \text {, }
$$

and since $\int_{0}^{1} f^{-}(u) d u \geq 1$, this shows that $y_{c, f}^{+}(u)$ reaches the value 1 in a finite time, so that no regular solutions to (7) will exist. 
(2) Second, we show that if (18) is violated, then no positive speeds will be admissible for our problem. Without loss of generality, we can assume that $\int_{0}^{1} f^{-}(u) d u<1$. Set $c=0$ and consider $y_{0, f}^{+}(\theta), y_{0, f}^{-}(\theta)$. It is clear that, since $\int_{0}^{1} f^{+}(u) d u \leq \int_{0}^{1} f^{-}(u) d u$,

$$
y_{0, f}^{+}(\theta)=\int_{0}^{\theta}-f(u) d u \geq y_{0, f}^{-}(\theta)=\int_{\theta}^{1} f(u) d u .
$$

If we now increase $c$, from $y_{0, f}^{-}(\theta)>0$ we infer, using Lemma 2.5, that for $c>0$ small we have

$$
y_{c, f}^{+}(\theta) \geq y_{0, f}^{+}(\theta) \geq y_{0, f}^{-}(\theta)>y_{c, f}^{-}(\theta) .
$$

Hence, using again Lemma 2.5, the two curves cannot meet for any value of $c>0$ and our problem does not have a solution.

(3) We finally show that if (18) and (19) hold, then there exists a unique admissible speed for $f$. To this aim, we focus again on $y_{c, f}^{+}(\theta)$ and $y_{c, f}^{-}(\theta)$.

Case 1. $\int_{\theta}^{1} f(u) d u<1$. Setting $\eta=\int_{0}^{1} f(u) d u$, from Lemma 2.4, together with the observation after Lemma 3.1, $y_{c, f}^{-}(\theta)$ and $y_{c, f}^{+}(\theta)$ are close respectively to

$$
\int_{\theta}^{1} f(u) d u=\int_{0}^{\theta}-f(u) d u+\eta \text { and } \int_{0}^{\theta}-f(u) d u
$$

for $c>0$ small. Since for $c$ large enough $y_{c, f}^{+}(\theta)$ reaches the value 1 , the result follows from continuity and monotonicity.

Case 2. $\int_{\theta}^{1} f(u) d u \geq 1$. Let $1>\xi_{0} \geq \theta$ be such that $\int_{\xi_{0}}^{1} f(u) d u=1$.

In order to apply the continuity argument, we claim that given any number $a<1$, there exists $\gamma>0$ such that the inequality $y_{c, f}^{-}(\theta) \geq a$ holds whenever $0<c<\gamma$. In fact, choose $\varepsilon$ such that

$$
1-\sqrt{\varepsilon}>a
$$

By continuous dependence, there exists $c_{0}>0$ such that if $0<c<c_{0}$ then $y_{c, f}^{-}(\xi) \geq 1-\sqrt{\varepsilon}$ for some $\xi>\xi_{0}$. The solution to

$$
w^{\prime}=c R(w), \quad w(\xi)=1-\sqrt{\varepsilon}
$$

is $w_{c}(s)=1-\sqrt{1-(k-c s)^{2}}$ with $k=c \xi+\sqrt{1-\varepsilon}$. Hence

$$
w_{c}(\theta)=1-\sqrt{1-(c(\xi-\theta)+\sqrt{1-\varepsilon})^{2}},
$$

so that $\liminf _{c \rightarrow 0} w_{c}(\theta) \geq 1-\sqrt{\varepsilon}$. On the other hand, since $f>0$ on $] \theta, 1[$, the graph of the solution $y_{c, f}^{-}$stays above the graph of $w_{c}$ in $] \theta, \xi$ [. Hence the claim follows and the proof is complete.

The picture highlighted in Proposition 3.9 is preserved up to changes in $f$ on a neighborhood of $\theta$, as shown by the following result of perturbative type. 
Proposition 3.10 Let $f$ be of type $C$ with $\eta=\int_{0}^{1} f(u) d u>0$, and $\int_{0}^{1} f^{+}(u) d u<1$. Let $G>2\|f\|_{\infty}$ be a given number. Take $\delta<\frac{1}{2 G} \min \left\{\eta, \int_{0}^{1} f^{-}(u) d u\right\}$ and let $\hat{f}$ be any continuous function that coincides with $f$ in $] 0, \theta-\delta[\cup] \theta+\delta, 1\left[\right.$, and satisfies $|\hat{f}(z)| \leq \frac{G}{2}$ for every $z \in[\theta-\delta, \theta+\delta]$. Then there exists a unique admissible speed for

$$
\left\{\begin{array}{l}
y^{\prime}=c \frac{\sqrt{y(2-y)}}{1-y}-\hat{f}(u), \\
y(0)=0=y(1) .
\end{array}\right.
$$

Proof Consider $y_{0, \hat{f}}^{+}$and $y_{0, \hat{f}}^{-}$. With the above notation, we have

$$
\left|y_{0, \hat{f}}^{+}(\theta)-y_{0, f}^{+}(\theta)\right|=\left|\int_{0}^{\theta}(\hat{f}(u)-f(u)) d u\right| \leq \delta G
$$

and, similarly,

$$
\left|y_{0, \hat{f}}^{-}(\theta)-y_{0, f}^{-}(\theta)\right|=\left|\int_{\theta}^{1}(\hat{f}(u)-f(u)) d u\right| \leq \delta G
$$

Therefore, by our choice of $\delta$, we have

$$
0<y_{0, f}^{+}(\theta)-\delta G<y_{0, \hat{f}}^{+}(\theta)<y_{0, f}^{+}(\theta)+\frac{\eta}{2}=y_{0, f}^{-}(\theta)-\frac{\eta}{2}<y_{0, \hat{f}}^{-}(\theta) .
$$

Hence, for $c>0$ sufficiently small we obtain

$$
0<y_{c, \hat{f}}^{+}(\theta)<y_{c, \hat{f}}^{-}(\theta)<1
$$

where we have used the arguments of Lemma 2.4 and Proposition 3.9, Step 3, Case 1. We can now repeat the previous reasonings to infer the existence of an admissible speed. The uniqueness follows again from Lemmas 2.5 and 3.8 .

It may be remarked that the two assumptions of Proposition 3.9 are satisfied for $\hat{f}$. Indeed, under the previous conditions we have

$$
\left|\int_{0}^{1}(f(u)-\hat{f}(u)) d u\right|<\eta
$$

so that, in particular, $\int_{0}^{1} \hat{f}(u) d u>\int_{0}^{1} f(u) d u-\eta=0$; on the other hand, $\hat{f}^{-}(u)=0$ for $u \in$ ]$\theta+\delta, 1[$, and in view of the choice of $\delta$,

$$
\int_{0}^{\theta+\delta} \hat{f}^{-}(u) d u<\int_{0}^{\theta-\delta} f^{-}(u) d u+\eta=\int_{\theta}^{1} f^{+}(u) d u-\int_{\theta-\delta}^{\theta} f^{-}(u) d u<1
$$

Turning to more general types of reaction, let us further observe that it suffices that there exists $0<u_{0}^{\prime} \leq u_{0}$ such that $f(u)<0$ for every $\left.u \in\right] 0, u_{0}^{\prime}\left[\right.$, with $f\left(u_{0}^{\prime}\right)=0$ (i.e., $f$ is negative in a neighborhood of 0 ) for the admissible speed (if any) to be unique, as it is possible to see with the usual monotonicity argument applied focusing on $y_{c, f}^{+}\left(u_{0}^{\prime}\right), y_{c, f}^{-}\left(u_{0}^{\prime}\right)$. However, in 


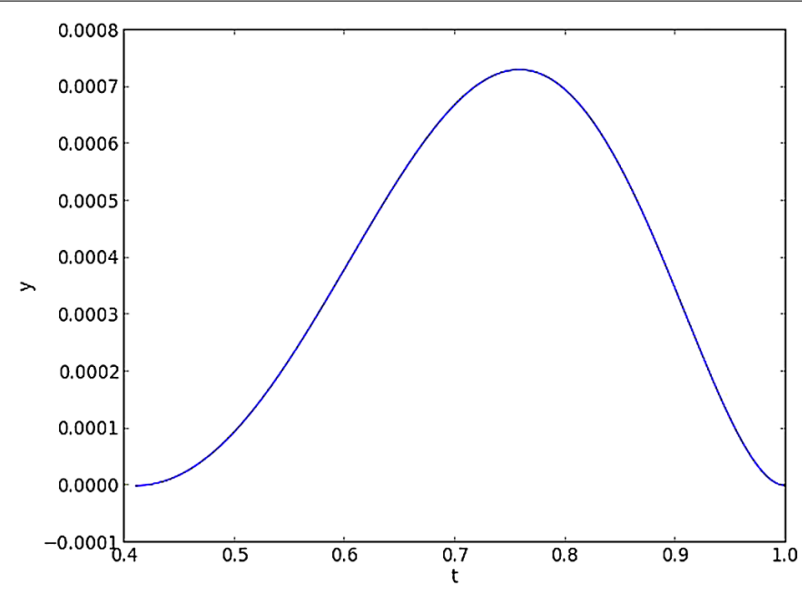

Figure 1 The solution to (8) for $f$ as in (20), with $c=0.0083516$.

this case it is not sufficient that (18) and (19) hold in order to have existence of an admissible speed, as the following example shows. Take

$$
f(u)=u(u-0.15)(0.41-u)(0.75-u)(1-u)
$$

in this case, $\int_{0}^{1} f(u) d u=0.000270833$ and (19) is shown to hold quite easily. However, we see that, for $c \geq \bar{c}=0.0083516$, the solution shot from the right vanishes in the interior of $[0,1]$, while $y_{c, f}^{-}(0)>0$ (see Figure 1 ) for $c<0.0083516$.

Indeed, the problem is that, on varying of $c, y_{c, f}^{-}$could vanish in ]0,1[ before the equality $y_{c, f}^{+}\left(u_{0}^{\prime}\right)=y_{c, f}^{-}\left(u_{0}^{\prime}\right)$ has been obtained. Of course, the situation is determined by the interplay between the intervals of positivity and negativity of $f$ and the corresponding values of the integral function: the farther it is from 0 , the better. In the next paragraph we will briefly go through a possible result in presence of more general reaction terms; as will easily be realized, a general analysis is far from being feasible.

\subsection{Remarks on more general reaction terms}

We briefly review a couple of situations for more general reaction terms.

Let $f \in C([0,1])$ satisfy (f) and denote by $0=\theta_{0}<\theta_{1}<\theta_{2}<\cdots<\theta_{r-1}<\theta_{r}=1$ its zeros $(r \geq 3)$. Write $\left.I_{j}=\right] \theta_{j-1}, \theta_{j}\left[, j=1, \ldots, r\right.$ and denote by $F_{0}, F_{1}$ the two integrals

$$
F_{0}(u)=\int_{0}^{u} f(\tau) d \tau, \quad F_{1}(u)=\int_{u}^{1} f(\tau) d \tau
$$

We first assume that each $\theta_{j}$ is simple, namely $f$ changes sign crossing $\theta_{j}, j=1, \ldots, r-1$. For such a reaction term, in order for solutions to (7) (with $c>0$ ) to exist, some conditions are necessary:

(a)

$$
\int_{I_{j}} f^{-}(u) d u<1
$$

for every $j=1, \ldots, r$. 
Indeed, if it were not the case, the solution $y_{c, f}^{-}(u)$ would vanish in $] 0,1[$, since, assuming that $\int_{I_{j^{\prime}}} f^{-}(u) d u \geq 1$ for a certain $j^{\prime}$, the solution to

$$
\left\{\begin{array}{l}
z^{\prime}=-f(t), \\
z\left(\theta_{j^{\prime}}\right)=y_{c, f}^{-}\left(\theta_{j^{\prime}}\right)<1
\end{array}\right.
$$

would be a (backward) supersolution for our problem, vanishing in a certain point of $I_{j^{\prime}}$.

Condition (21) is necessary also in order to avoid that the solutions $y_{c, f}^{+}$shot forward touch the barrier $y=1$ (both in the case $f$ is positive in a neighborhood of 0 , shooting from $\theta_{1}$, and if $f$ is negative in a neighborhood of 0 , shooting from $\theta_{0}$ ).

According to the terminology of Section 5 below, we are avoiding the appearance of discontinuous steady states.

(b)

$$
F_{1}(u)>0 \quad \text { for every } u \in[0,1]
$$

In particular, a necessary condition is that $\int_{0}^{1} f(u) d u>0$.

These conditions are not sufficient, in general, for the existence of an admissible speed. Nevertheless, we can prove the existence of an admissible speed for a quite general class of functions generalizing type $\mathrm{C}$.

Proposition 3.11 Let $f$ satisfy the assumptions above, with $f(u)<0$ on $I_{1}$. Assume that $\left.\left.t_{0} \in\right] 0,1\left[, t_{1} \in\right] 0, \theta_{r-1}\right]$ are such that

$$
\left.F_{0}(t)<0 \text { for every } t \in\right] 0, t_{0}\left[\text { and } F_{0}\left(t_{0}\right)=\min _{t \in\left[0, t_{0}\right]} F_{0}(t)\right. \text {, }
$$

and

$$
F_{1}\left(t_{1}\right)=\min _{t \in\left[t_{1}, \theta_{r-1}\right]} F_{1}(t)
$$

If $t_{0} \geq t_{1}$, then there exists a unique admissible speed for $f$.

Proof We focus on $y_{c, f}^{+}\left(t_{0}\right), y_{c, f}^{-}\left(t_{0}\right)$. In view of the sign assumption in (22), for every $c \geq 0$ we have that

$$
y_{c, f}^{+}(u)>0
$$

for every $\left.u \in] 0, t_{0}\right]$, since for $c=0$ we have a positive subsolution, and the solution shot from the left is unique thanks to the sign assumption in a right neighborhood of 0 . On the other hand, the positivity of $\int_{0}^{1} f(u) d u$ guarantees that, for every $\left.t \in\right] 0,1[$,

$$
y_{0, f}^{-}(t)=\int_{t}^{1} f(u) d u>-\int_{0}^{t} f(u) d u=y_{0, f}^{+}(t)
$$

Increasing the value of $c$, we can conclude using the standard monotonicity and continuous dependence argument for $t=t_{0}$ if we are able to ensure that $y_{c, f}^{+}$does not reach the 
barrier $y=1$ in $] 0, t_{0}\left[\right.$ and $y_{c, f}^{-}$does not vanish in $] t_{0}, 1\left[\right.$ (notice that, in any case, $y_{c, f}^{-}(u)$ cannot vanish for $u \in] \theta_{r-1}, 1$ [, as a direct consequence of the sign of the derivative). However, (22) gives, for every $u \in] 0, t_{0}[$

$$
\begin{aligned}
y_{c, f}^{+}\left(t_{0}\right)-y_{c, f}^{+}(u) & =\int_{u}^{t_{0}} c \frac{\sqrt{y_{c, f}^{+}(\tau)\left(2-y_{c, f}^{+}(\tau)\right)}}{1-y_{c, f}^{+}(\tau)} d \tau-\int_{u}^{t_{0}} f(\tau) d \tau \\
& \geq F_{0}(u)-F_{0}\left(t_{0}\right)>0 .
\end{aligned}
$$

Similarly, assumption (23) allows to claim that, for every $u \in] t_{1}, \theta_{r-1}[$,

$$
y_{c, f}^{-}\left(t_{1}\right)-y_{c, f}^{-}(u)=\int_{t_{1}}^{u} f(\tau) d \tau-c \int_{t_{1}}^{u} \frac{\sqrt{y_{c, f}^{-}(\tau)\left(2-y_{c, f}^{-}(\tau)\right)}}{1-y_{c, f}^{-}(\tau)} d \tau \leq F_{1}\left(t_{1}\right)-F_{1}(u)<0 .
$$

The statement now follows noticing that, in view of (24), $y_{0, f}^{-}(u)>y_{0, f}^{+}(u)$ for every $u \in$ $\left[t_{1}, t_{0}\right]$. Hence, using Lemmas 2.5 and 3.8, on growing of $c>0$ we can invoke the usual continuous dependence and monotonicity argument thanks to (25) and (26), which guarantee that both $y_{c, f}^{+}$cannot reach 1 and $y_{c, f}^{-}$cannot vanish without crossing each other.

As an example, we can take $f$ given by (see Figure 2)

$$
f(u)=u(u-0.2)(u-0.35)(u-0.7)(1-u)
$$

the plots of $f(u), F_{0}(u)$, and $F_{1}(u)$ are represented in Figure 3.

Choosing $t_{0}, t_{1}$ in a sufficiently small neighborhood of 0 (for instance $t_{0}=t_{1}=0.04$ ), all the assumptions of Proposition 3.11 are satisfied. The numerical simulations show the
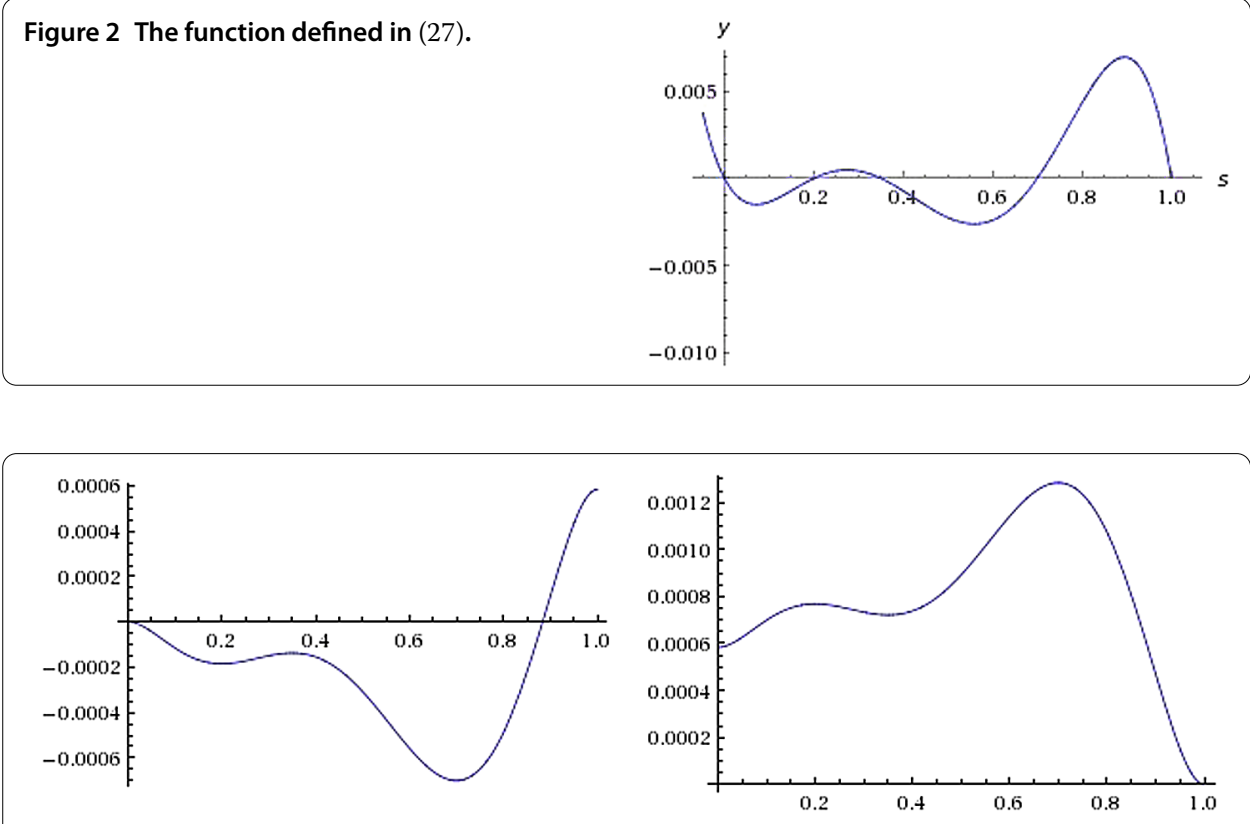

Figure 3 The primitives $F_{0}(u)$ (on the left) and $F_{1}(u)$ (on the right). 


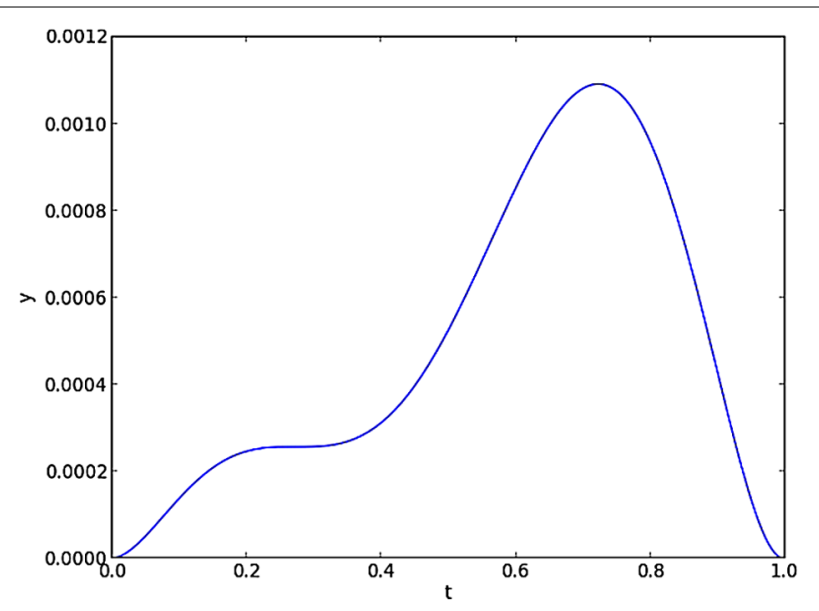

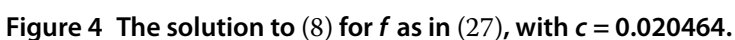

existence of an admissible speed which is approximately equal to 0.020464 , as shown in Figure 4.

On the other hand, a comment on the situation where $f$ is non-negative and has a multiple zero in ]0,1[ may be of interest. In this case, the usual monotonicity argument does not work and other kinds of phenomena are possible, in principle. For instance, given $\alpha \in] 0,1[$ consider the function

$$
f(u)=u(1-u)(u-\alpha)^{2} .
$$

For this reaction term, the solution shot from the right (which is unique) is always nonnegative (since 0 is a subsolution) and may vanish only in $\alpha$ or in 0 . We have the following picture:

- for small values of $c$, we have $y_{c, f}^{-}(\alpha)>0, y_{c, f}^{-}(0)>0$;

- for large values of $c$, the situation, restricted to $[\alpha, 1]$, is similar to the type A case, so that we have a heteroclinic connection between 1 and $\alpha$.

Thus, the admissible speeds for a connection between 1 and 0 (if any) form a bounded interval $\left[c_{1}, c_{2}[\right.$, open on the right, while the connections between 1 and $\alpha$ appear for $c \in\left[c_{2},+\infty\right.$ [. Also, at least for $c \geq c_{1}$, there appear connections between $\alpha$ and 0 , as well (otherwise the solution shot from $\alpha$ would intersect the one shot from 1 , which is impossible). It is clear that the possibility of having a connection between 1 and 0 is strictly related to the position of $\alpha$. In particular, if, with the same notation as before,

$$
F_{1}(\alpha) \approx 0
$$

i.e., $\alpha \approx 1$, then it is likely that we will not have a connection between 0 and 1 , since, as $c$ increases, $y_{c, f}^{-}(\alpha)$ will approach 0 faster than $y_{c, f}^{-}(0)$. This is the case, for instance, when $\alpha=0.9$ : the simulations show that, passing from $c_{1}=0.063031568$ to $c_{2}=0.063031569$, we have $\min \left\{y_{c_{1}, f}^{-}(0), y_{c_{1}, f}^{-}(\alpha)\right\}>0$ and $y_{c_{2}, f}^{-}(\alpha)=0$ (see Figures 5 and 6).

Just to make the 'opposite' example, if $\alpha=0.1$, e.g., we expect the existence of admissible speeds for a connection between 1 and 0 , and indeed the numerical simulations show an 


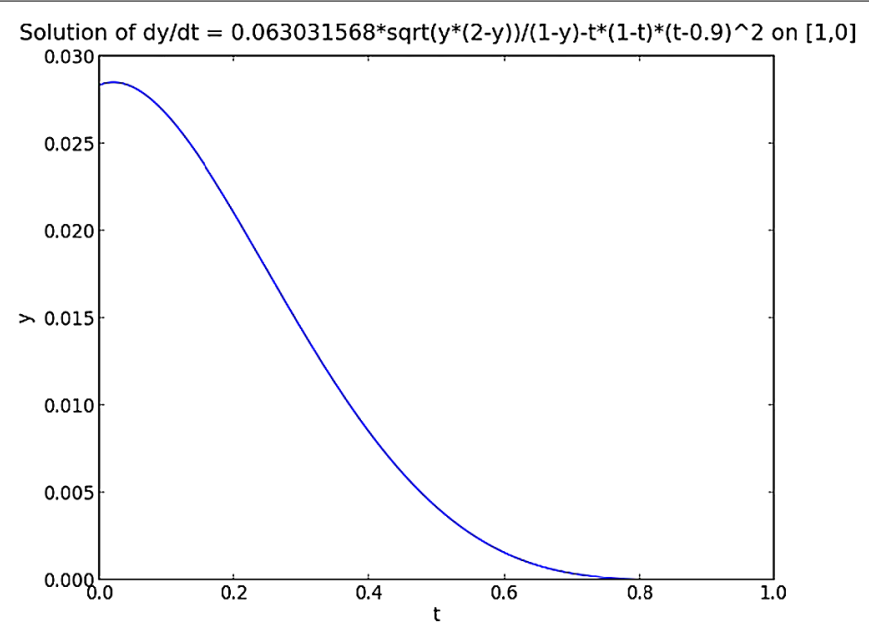

Figure 5 The solution to (8) for $f$ as in (28), with $\alpha=0.9$, and $c=0.063031568$.

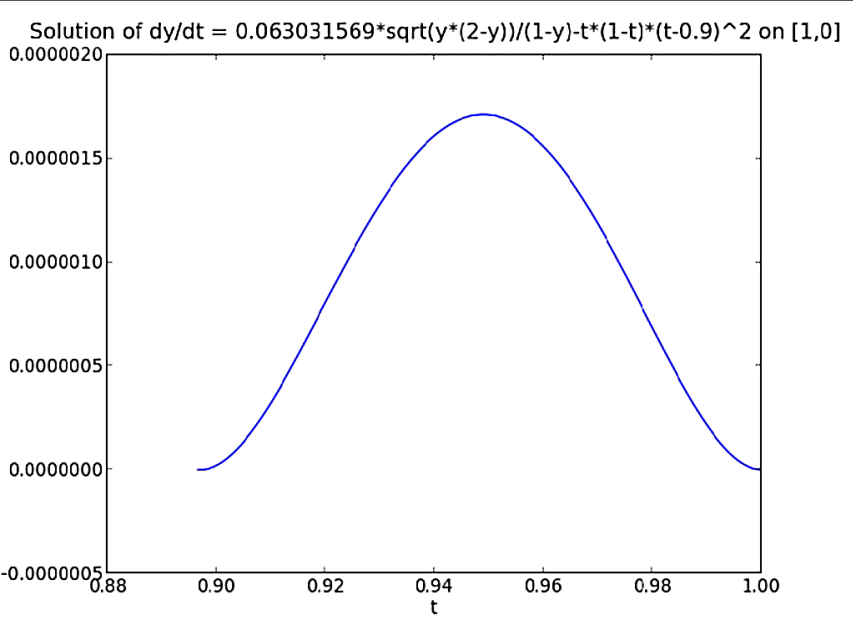

Figure 6 The solution to (8) for $f$ as in (28), with $\alpha=0.9$, and $c=0.063031569$.

interval of such admissible speeds approximately equal to [0.366, 0.419[, threshold from which on there exist connections between 1 and 0.1 (see Figures 7, 8, and 9).

Our equation seems to be quite sensitive to large values of the parameter, so that the reliability of the simulations should be considered with caution.

The variety of configurations which may appear suggests that a general analysis is far from being feasible without the expense of heavy assumptions.

\section{A mechanical interpretation: a quasilinear damped pendulum}

In this section we give a motivation to the second order equation (5) and the problem (7) that does not come from (2).

To this end, notice that, taking $f$ as a sinusoidal function, the equation

$$
\left(\frac{u^{\prime}}{\sqrt{1+u^{\prime 2}}}\right)^{\prime}-c u^{\prime}+B \sin u=0
$$




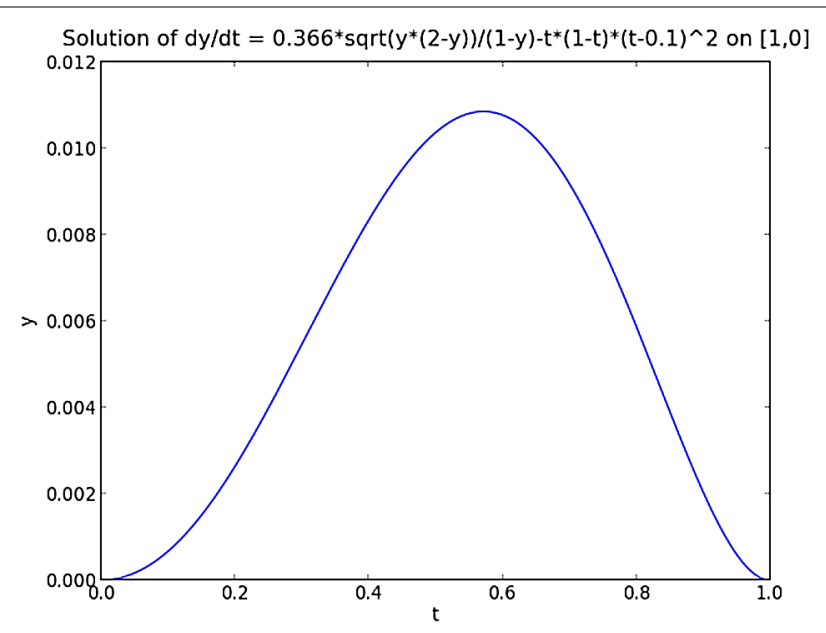

Figure 7 The solution to (8) for $f$ as in (28), with $\alpha=0.1$, and $c=0.366$.

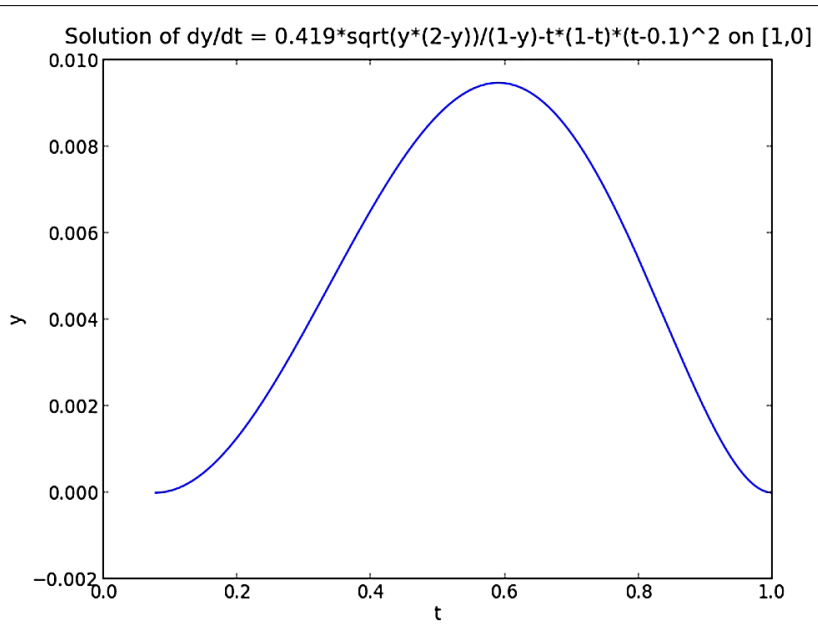

Figure 8 The solution to (8) for $f$ as in (28), with $\alpha=0.1$, and $c=0.419$ : from this value of $c$ on, the solution $y_{c, f}^{-}$starts vanishing at $\alpha$.

can be seen to describe the motion of a quasilinear pendulum with constant damping, where the mean curvature operator is used to define acceleration; in the undamped case, (29) has been recently studied by several authors $[7,11,25,26]$. The results obtained in the previous sections can be reinterpreted in this direction, the admissible speeds corresponding to suitable values of the damping that lead to the existence of a monotone motion between two equilibria. Throughout the paragraph, the word branch refers to the first order model associated with (29).

In fact, we will take into account a slightly more general equation, admitting the presence of an external constant torque $D>0$ and considering, as usual, a positive damping. This leads to the following quasilinear pendulum equation:

$$
\left(\frac{u^{\prime}}{\sqrt{1+u^{\prime 2}}}\right)^{\prime}+A u^{\prime}+B \sin u-D=0, \quad A, B, D>0
$$




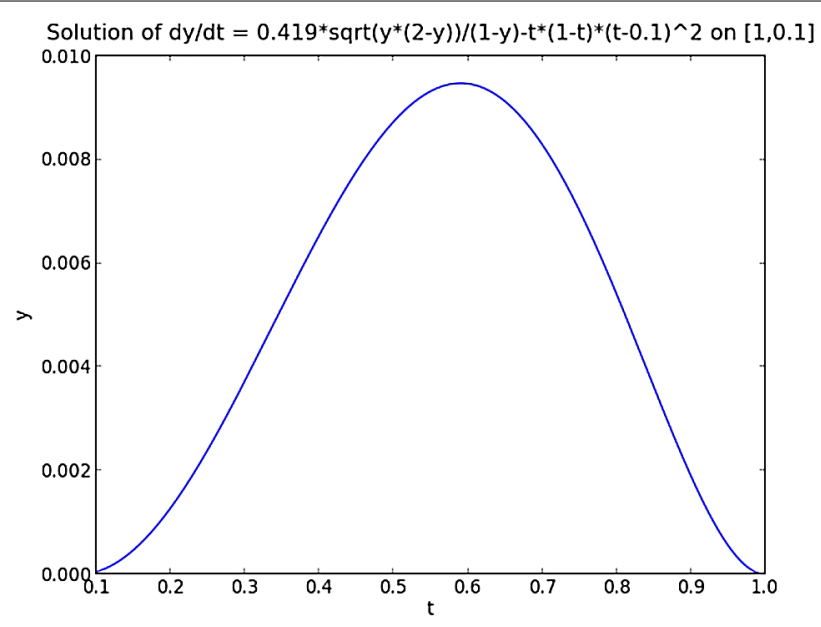

Figure 9 The rising of a connection between 0.1 and 1 , for $f$ as in (28), with $\alpha=0.1$, and $c=0.419$.

Let us just remark that the 'bridge' between this problem and the corresponding one with positive parameter $c$ is given, at the first order level, by introducing new variables, say $z(u)=y(k-u)$, so that the equilibria are reversed.

A numerical approach to the analogous classical pendulum equation was proposed in [27].

In a $2 \pi$-period, we can isolate, in general, a stable equilibrium and an unstable one, which repeat themselves by $2 \pi$-periodicity:

$$
u_{S}=\arcsin \frac{D}{B} \in[0, \pi / 2], \quad u_{U}=\pi-\arcsin \frac{D}{B} \in[\pi / 2, \pi] .
$$

If $D>B$, no equilibrium exists; indeed, the torque is high enough for the motion to be a rotation. Of course, the solution to the first order model can reach the threshold 1 in a finite time (for instance if the torque is sufficiently high). On the other hand, if $D=B$ then the two equilibria collapse into the unique unstable one $\bar{u}=\pi / 2$. This is similar to a type A setting, with reversed signs: in particular, there exists a value $A^{*}>0$ such that for every $A \geq A^{*}$ the solutions to (30) are increasing heteroclinics between $\pi / 2$ and $5 \pi / 2$, otherwise they are rotations which globally exist forward in time.

Now consider the dynamics for smaller values of the torque $(D<B)$, taking into account the existence of solutions going monotonically to an equilibrium and (without loss of generality because of periodicity) restricting our interest to the intervals $\left[u_{S}, u_{S}+2 \pi\right]$ or $\left[u_{U}, u_{U}+2 \pi\right]$.

Assume first $D=0$ and consider the solutions starting from (or arriving at) the unstable equilibrium. A first difference, compared with the classical damped pendulum equation $u^{\prime \prime}+A u^{\prime}+B \sin u=0$, is that the (unique) solution $\bar{y}(u)$ to the associated first order model, approaching $u_{U}$ in an increasing way as $t \rightarrow+\infty$, may not be global. In fact, on the one hand the first order problem (where $y=u^{\prime 2}$ )

$$
\left\{\begin{array}{l}
y^{\prime}=-2(A \sqrt{y}+B \sin u), \\
y(\pi)=0, \quad y(u)>0 \quad \text { for } u \neq \pi,
\end{array}\right.
$$


has the nonnegative lower solution $y(u)=2 B(1+\cos u)$, so that $\bar{y}(u)$ is always defined and increasing and corresponds to a rotation type motion. On the other hand, the presence of the singularity for $y=1$ makes the curvature case slightly different: indeed, we always have backward and forward local existence and uniqueness for

$$
\left\{\begin{array}{l}
y^{\prime}=-A \frac{\sqrt{y(2-y)}}{1-y}-B \sin u, \\
y(\pi)=0, \quad y(u)>0 \quad \text { for } u \neq \pi
\end{array}\right.
$$

where $y=Q\left(u^{\prime}\right)$ (since, respectively, $-\sin u$ is negative in a left neighborhood of $\pi$, and $-A R(y)$ is monotone decreasing), but the solution $\hat{y}(u)$ to (31) blows up in a finite backward time. This is easily seen in view of the fact that $\hat{y}(u)$ is always positive, so that, for every integer $k$,

$$
y_{-A, f}^{-}(-(2 k+1) \pi)-y_{-A, f}^{-}(-(2 k-1) \pi)=\int_{-(2 k+1) \pi}^{-(2 k-1) \pi} A \frac{\sqrt{y_{-A, f}^{-}\left(2-y_{-A, f}^{-}\right)}}{1-y_{-A, f}^{-}} d u
$$

since $y_{-A, f}^{-}(-3 \pi)=: \alpha_{c}>0$, the value of $\hat{y}(u)$ increases by a quantity which is bounded away from 0 as $u$ decreases by a multiple of $2 \pi$, eventually reaching 1 .

Looking forward in time, on the contrary, the existence and uniqueness for (31) ensure that there is an increasing solution starting at $\pi$ for $t \rightarrow-\infty$, whose behavior is determined by the value of $A$ (taken into account that $y_{0}(u)=B+B \cos u$ is a forward upper solution which vanishes at $u=3 \pi)$. In particular, there exists a value $A^{*}>0$ such that

- if $A \geq A^{*}$, then the solution is an increasing connection between $\pi$ and $2 \pi$;

- if $A<A^{*}$, then the solution oscillates around the stable equilibrium $2 \pi$, since by strict monotonicity it vanishes before reaching $3 \pi$ (for $c=0$ one has $y_{0, f}(3 \pi)=0$ ).

More generally, we have the following counterpart of Proposition 3.2.

Proposition 4.1 Let $D \geq 0$. There exists $A_{D}^{*}>0$ such that the problem

$$
\left\{\begin{array}{l}
y^{\prime}=-A \frac{\sqrt{y(2-y)}}{1-y}-B \sin u+D, \\
\left.y\left(u_{U}\right)=0=y\left(u_{S}+2 \pi\right), \quad y(u)>0 \quad \text { for } u \in\right] u_{U}, u_{S}+2 \pi[
\end{array}\right.
$$

has a solution if and only if $A \geq A_{D}^{*}$.

It turns out that

the critical value $A_{D}^{*}$ assumes the meaning of a threshold damping,

discriminating between different kinds of motions. In the preceding considerations we have $A^{*}=A_{0}^{*}$. Via an analogue of Lemma 3.1 and Proposition 3.2, the fact that $\sin u \leq u \leq$ $u /\left(1-u^{2}\right)$ implies that $A_{0}^{*}=2 \sqrt{B}$.

It may be added that, in the absence of torque, for $A \geq A_{0}^{*}$ the motion that starts from a rest position at $\left.u_{0} \in\right] \pi, 2 \pi$ [ ends monotonically at $2 \pi$ as time goes to infinity. On the other hand, if $A<A_{0}^{*}$ such motions approach $2 \pi$ in an oscillatory manner. 
In a similar way, the characterization of the possible motions for $D>0$ is now clear:

(a) If $A<A_{D}^{*}$, then

- the branch shot forward from $u_{U}$, which does not reach the threshold 1 in view of the sign of the derivative and stays over the connection $y_{A_{D}^{*}}$ by monotonicity (thus existing globally) is either a positive, rotational motion (if $A$ is small: the torque wins over damping), or it vanishes at some point inside the interval $] u_{S}+2 \pi, u_{U}+2 \pi[$, being an oscillation around the stable equilibrium $u_{S}+2 \pi$;

- the branch shot backward from $u_{S}+2 \pi$ vanishes before reaching $u_{U}$, thus giving rise to an oscillatory type motion (the less the value of the damping, the nearer the vanishing point will be to $u_{S}+2 \pi$ ).

(b) If $A \geq A_{D}^{*}$, the damping is too high to climb over $u_{S}+2 \pi$, and, on the other hand, the solution to the first order model cannot stop out of the equilibrium, so that we find the already mentioned increasing connections between $u_{U}$ and $u_{S}+2 \pi$.

\section{Discontinuous steady states}

In [9], the existence of traveling waves for reaction terms of type $C$ was related with the appearance of discontinuous steady states. In this last section, we want to briefly look at our first order model from this perspective.

As a first trivial remark, notice that if we restricted our attention to continuous steady states, i.e., classical solutions to

$$
\left\{\begin{array}{l}
y^{\prime}=-f(u), \\
y(0)=0=y(1),
\end{array}\right.
$$

taking values strictly between 0 and 1 , then they can obviously exist if and only if $\int_{0}^{1} f(u) d u=0$, so the problem is immediately solved in this case (hence excluding the cases $f \in \mathcal{A}$ and $f \in \mathcal{B}$ ). On the other hand, the cases $f \in \mathcal{A}$ and $f \in \mathcal{B}$ are dropped out also from the search for discontinuous steady states in any reasonable sense, since in the first situation the solution from the left goes negative, while in the second it remains constantly zero for a certain time interval. Therefore we restrict our interest to type $\mathrm{C}$ functions.

Of course, the solutions to $y^{\prime}=-f(u)$ from the left and from the right are unique.

Definition 5.1 Let $f \in \mathcal{C}$. We will say that $y(u)$ is a discontinuous steady state for (32) if there exist $\left.\theta_{1}<\theta_{2} \in\right] 0,1$ [, with $\theta_{1} \leq \theta$ and $\theta_{2} \geq \theta$, such that $y$ is defined and continuous in $\left[0, \theta_{1}\right] \cup\left[\theta_{2}, 1\right], y(u)$ satisfies $(32)$ in $\left[0, \theta_{1}[\cup] \theta_{2}, 1\right]$ and $y\left(\theta_{1}\right)=y\left(\theta_{2}\right)=1$. If such conditions are verified for $\theta_{1}=\theta_{2}$, we will say that $y(u)$ is a border steady state.

The definition corresponds to heteroclinic connections between 0 and 1 which 'break' at some time instant; with the notation of the previous section, this means that both $y_{0, f}^{+}$ and $y_{0, f}^{-}$reach the value 1 in a finite time. For a border steady state, coming back to the second order model, we would have a continuous heteroclinic connection between 0 and 1 having infinite derivative in a point.

Indeed, it is worth observing that the adequate variational setting for the second order equation

$$
\left(\frac{u^{\prime}}{\sqrt{1+u^{\prime 2}}}\right)^{\prime}=-f(u)
$$


is the space of bounded variation functions. As observed in [6, Remark 1.1], a discontinuous steady state is a weak solution (in the $B V$-sense) to (33), according to [6, Definition 1.1]. In such a kind of solution, a subshock appears: since $y(u)=1-1 / \sqrt{1+u^{\prime}(t(u))^{2}}$, whenever $y(u)=1$ we have $u^{\prime}(t(u))=+\infty$. Of course, the subshock solution will be defined up to an interval, at whose endpoints the derivative (which is infinite) must have the same sign in order for the speed of the subshock to be 0 (via an analogous of the Rankine-Hugoniot condition). If one dropped this requirement, there could be other kinds of solutions solving (33) almost everywhere, not fulfilling anyway any condition at the jump. We will not investigate further the existence of such pseudo-solutions.

We now show the validity of the following proposition, agreeing with [9].

Proposition 5.2 If $f \in \mathcal{C}$, there occurs one and only one of the following alternatives:

(1) there exists a discontinuous steady state for (32);

(2) there exists an admissible speed $c \in \mathbb{R}$ for (7).

Proof As usual, we write $f(u)=f^{+}(u)-f^{-}(u)$, with $f^{+}(u)=\max \{f(u), 0\}$ and $f^{-}(u)=$ $\max \{-f(u), 0\}$.

Assume first that $\int_{0}^{1} f(u) d u>0$, namely $\int_{0}^{1} f^{+}(u) d u>\int_{0}^{1} f^{-}(u) d u$.

Case 1: $\int_{0}^{1} f^{-}(u) d u<1$. Then, as we have seen, there exists a unique positive admissible speed. Also, no discontinuous steady states appear, since the solution $y_{0, f}^{+}$shot from the left cannot reach 1.

Case 2: $\int_{0}^{1} f^{-}(u) d u \geq 1$. As we have seen, no traveling waves with positive speed appear. On the other hand, the integral of $f^{+}$is strictly greater than 1 , so that there exist $\theta_{1}<$ $\theta_{2}$ with $y_{0, f}^{+}\left(\theta_{1}\right)=1=y_{0, f}^{-}\left(\theta_{2}\right)$, yielding the discontinuous steady state. Finally, no traveling waves with negative speed are possible, as well, since by monotonicity $y_{c, f}^{-}$would reach the threshold 1 all the same in a finite time.

Suppose next that $\int_{0}^{1} f(u) d u<0$, so that $\int_{0}^{1} f^{+}(u) d u<\int_{0}^{1} f^{-}(u) d u$. Setting $g(u)=-f(1-u)$ one has $g \in \mathcal{C}, \int_{0}^{1} g(u) d u=-\int_{0}^{1} f(u) d u>0$ and

$$
\int_{0}^{1} g^{+}(u) d u=\int_{0}^{1} f^{-}(u) d u>\int_{0}^{1} f^{+}(u) d u=\int_{0}^{1} g^{-}(u) d u,
$$

so that we are led back to the previous arguments. In particular, if $\int_{0}^{1} f^{+}(u) d u \geq 1$, a discontinuous steady state appears. Otherwise there exists a positive admissible speed for $g$, i.e., a solution with $c=c_{0}>0$ to

$$
\left(\frac{u^{\prime}}{\sqrt{1+u^{\prime 2}}}\right)^{\prime}-c u^{\prime}-f(1-u)=0
$$

setting $w=1-u$, it follows that $-c_{0}$ is a negative admissible speed for the existence of an increasing solution to the original problem

$$
\left(\frac{w^{\prime}}{\sqrt{1+w^{\prime 2}}}\right)^{\prime}-c w^{\prime}+f(w)=0
$$

Finally, assume $\int_{0}^{1} f(u) d u=0$, i.e., $\int_{0}^{1} f^{-}(u) d u=\int_{0}^{1} f^{+}(u) d u$.

Case 1: $\int_{0}^{1} f^{-}(u) d u<1$. A continuous steady state (or a traveling wave with $c=0$ ) appears. 
Case 2: $\int_{0}^{1} f^{-}(u) d u \geq 1$. We have a border steady state (if equality holds) or a discontinuous one (if the inequality is strict).

Let us give, as an example, the case when

$$
f(u)=-\beta^{2} u(u-\alpha)(u-1)
$$

for $\alpha \in] 0,1[$ and $\beta \neq 0$, treated extensively in [9] (notice that the notation used therein has the opposite sign both in the equation and in the function). For such a choice, it is

$$
\begin{aligned}
\int_{0}^{1} f(u) d u & =\frac{1-2 \alpha}{12}, \quad \int_{0}^{1} f^{-}(u) d u=\frac{(2-\alpha) \alpha^{3}}{12} \\
\int_{0}^{1} f^{+}(u) d u & =\frac{1-2 \alpha+2 \alpha^{3}-\alpha^{4}}{12} .
\end{aligned}
$$

We are going to recover the already known picture for this kind of function. As a preliminary observation, notice that if $\alpha=1 / 2$, namely $\int_{0}^{1} f(u) d u=0$, then we have a continuous steady state if $\beta^{2} \frac{(2-\alpha) \alpha^{3}}{12}=\beta^{2} \frac{1-2 \alpha+2 \alpha^{3}-\alpha^{4}}{12}<1$, or a discontinuous one if this condition is violated. We now turn to the other cases:

(1) $\alpha<1 / 2$. In this setting, we have $\int_{0}^{1} f(u) d u>0$, so that two possibilities can occur:

- if $\beta^{2} \frac{(2-\alpha) \alpha^{3}}{12}<1$, we use the first part of the proof of Proposition 5.2, Case 1, to infer the existence of a traveling wave with positive speed;

- if $\beta^{2} \frac{(2-\alpha) \alpha^{3}}{12} \geq 1$, we find a discontinuous steady state as a consequence of Case 2 in the first part of the proof of Proposition 5.2.

(2) $\alpha>1 / 2$. We now have $\int_{0}^{1} f(u) d u<0$, so that the following situations are easily shown to hold as a consequence of the second part of the proof of Proposition 5.2:

- if $\beta^{2} \frac{1-2 \alpha+2 \alpha^{3}-\alpha^{4}}{12}<1$, we find a traveling wave with negative speed;

- if $\beta^{2} \frac{1-2 \alpha+2 \alpha^{3}-\alpha^{4}}{12} \geq 1$, then a discontinuous steady state emerges.

This picture perfectly agrees with [9, Section 2.2 and Figure 2].

We finally remark that, in the case of a more general type of reaction like the one discussed in Section 3.4, nonexistence of discontinuous steady states together with nonexistence of admissible speeds could occur, since the solution shot from the right can vanish before the admissible speed has been reached.

\section{Competing interests}

The authors declare that they have no competing interests.

Authors' contributions

All authors contributed equally to the writing of this paper. All authors read and approved the final manuscript.

\section{Author details}

${ }^{1}$ Dipartimento di Matematica ed Applicazioni, Università di Milano-Bicocca, Via Cozzi, 55, Milano, 20125, Italy. ${ }^{2}$ CMAF,

Faculdade de Ciências da Universidade de Lisboa, Avenida Professor Gama Pinto, 2, Lisboa, 1649-003, Portugal.

\section{Acknowledgements}

The first author wishes to acknowledge the support of the Project PTDC/MAT/113383/2009 during his stay in Lisbon. The second author is supported by Fundação para a Ciência e a Tecnologia, PEst-OE/MAT/UI0209/2013. 


\section{References}

1. Kolmogorov, A, Petrovsky, I, Piskounov, N: Étude de l'équation de la diffusion avec croissance de la quantité de matière et son application à un problème biologique. Mosc. Univ. Math. Bull. 1, 1-25 (1937)

2. Maini, PK, Malaguti, L, Marcelli, C, Matucci, S: Diffusion-aggregation processes with mono-stable reaction terms. Discrete Contin. Dyn. Syst., Ser. B 6, 1175-1189 (2006)

3. Fife, PC, Mc Leod, JB: The approach of solutions of nonlinear diffusion equations to travelling front solutions. Arch. Ration. Mech. Anal. 65, 335-361 (1977)

4. Fife, PC, MC Leod, JB: A phase plane discussion of convergence to travelling fronts for nonlinear diffusion. Arch. Ration. Mech. Anal. 75, 281-314 (1980/81)

5. Rosenau, P: Tempered diffusion: a transport process with propagating front and inertial delay. Phys. Rev. A 46, 7371-7374 (1992)

6. Bonheure, D, Obersnel, F, Omari, P: Heteroclinic solutions of the prescribed curvature equation with a double-well potential. Differ. Integral Equ. 26, 1411-1428 (2013)

7. Cid, JA, Torres, PJ: On the existence and stability of periodic solutions for pendulum-like equations with friction and $\phi$-Laplacian. Discrete Contin. Dyn. Syst., Ser. A 33, 141-152 (2013)

8. Bonheure, D, Habets, P, Obersnel, F, Omari, P: Classical and non-classical solutions of a prescribed curvature equation. J. Differ. Equ. 243, 208-237 (2007)

9. Kurganov, A, Rosenau, P: On reaction processes with saturating diffusion. Nonlinearity 19, 171-193 (2006)

10. Benevieri, $\mathrm{P}$, do Ó, JM, de Medeiros, ES: Periodic solutions for nonlinear equations with mean curvature-like operators, Appl. Math. Lett. 20, 484-492 (2007)

11. Bereanu, C, Jebelean, $P$, Mawhin, J: Periodic solutions of pendulum-like perturbations of singular and bounded $\phi$-Laplacians. J. Dyn. Differ. Equ. 22, 463-471 (2010)

12. Habets, $\mathrm{P}$, Omari, P: Multiple positive solutions of a one-dimensional prescribed mean curvature problem. Commun. Contemp. Math. 9, 701-730 (2007)

13. Obersnel, F, Omari, P: Positive solutions of the Dirichlet problem for the prescribed mean curvature equation. J. Differ. Equ. 249, 1674-1725 (2010)

14. Obersnel, F, Omari, P: Multiple bounded variation solutions of a periodically perturbed sine-curvature equation. Commun. Contemp. Math. 13, 863-883 (2011)

15. Calvo, J, Campos, J, Caselles, V, Sánchez, O, Soler, J: Pattern formation in a flux limited reaction-diffusion equation of porous media type, preprint

16. Campos, J, Guerrero, P, Ó Sánchez, Soler, J: On the analysis of travelling waves to a nonlinear flux limited reaction-diffusion equation. Ann. Inst. Henri Poincaré, Anal. Non Linéaire 30(1), 141-155 (2013)

17. Malaguti, L, Marcelli, C: Travelling wavefronts in reaction-diffusion equations with convection effects and non-regular terms. Math. Nachr. 242, 148-164 (2002)

18. Berestycki, H, Nirenberg, L: Travelling fronts in cylinders. Ann. Henri Poincaré 9, $497-572$ (1992)

19. Aronson, DG, Weinberger, HF: Multidimensional nonlinear diffusion arising in population genetics. Adv. Math. 30 , 33-76 (1978)

20. Sennings, JR: First order differential equation solver, website. http://www.math-cs.gordon.edu/ senning/software.html

21. Coelho, I, Sanchez, L: Travelling wave profiles in some models with nonlinear diffusion. Appl. Math. Comput. 235, 469-481 (2014)

22. Correia, S, Sanchez, L: Ondas progressivas no modelo de Fisher-Kolmogorov - um clássico moderno. Bol. Soc. Port. Mat. 67, 165-184 (2012)

23. Enquiça, R, Gavioli, A, Sanchez, L: A class of singular first order differential equations with applications in reaction-diffusion. Discrete Contin. Dyn. Syst., Ser. A 33, 173-191 (2013)

24. Bonheure, D, Sanchez, L: Heteroclinic orbits for some classes of second and fourth order differential equations. In: Cañada, A, Drábek, P, Fonda, A (eds.) Handbook of Differential Equations: Ordinary Differential Equations, vol. 3. Elsevier, Amsterdam (2006)

25. Bereanu, C, Mawhin, J: Periodic solutions of nonlinear perturbations of $\phi$-Laplacians with possibly bounded $\phi$. Nonlinear Anal. 68, 1668-1681 (2008)

26. Obersnel, F, Omari, P, Rivetti, S: Existence, regularity and stability properties of periodic solutions of a capillarity equation in the presence of lower and upper solutions. Nonlinear Anal., Real World Appl. 13, 2830-2852 (2012)

27. Falco, CM: Phasespace of a driven, damped pendulum (Josephson weak link). Am. J. Phys. 44, 733-740 (1976)

\section{Submit your manuscript to a SpringerOpen ${ }^{\odot}$ journal and benefit from:}

- Convenient online submission

Rigorous peer review

- Immediate publication on acceptance

- Open access: articles freely available online

- High visibility within the field

- Retaining the copyright to your article 